\title{
Metabolite Profiling of Triterpene Saponins in Medicago truncatula Hairy Roots by Liquid Chromatography Fourier Transform Ion Cyclotron Resonance Mass Spectrometry
}

\author{
Jacob Pollier, ${ }^{+, \neq}$Kris Morreel, ${ }^{+, \neq}$Danny Geelen, ${ }^{\S}$ and Alain Goossens ${ }^{*,+, \neq}$ \\ ${ }^{\dagger}$ Department of Plant Systems Biology, VIB, B-9052 Gent, Belgium \\ ${ }^{\ddagger}$ Department of Plant Biotechnology and Genetics, Ghent University, B-9052 Gent, Belgium \\ ${ }^{\S}$ Department of Plant Production, Faculty of Bioscience Engineering, Ghent University, B-9000 Gent, Belgium
}

\begin{abstract}
Triterpenes are one of the largest classes of plant natural products, with an enormous variety in structure and bioactivities. Here, triterpene saponins from hairy roots of the model legume Medicago truncatula were profiled with reversedphase liquid chromatography coupled to negative-ion electrospray ionization Fourier transform ion cyclotron resonance mass spectrometry (LC ESI FT-ICR MS). Owing to the accuracy of the FT-ICR MS, reliable molecular formulas of the detected compounds could be predicted, which, together

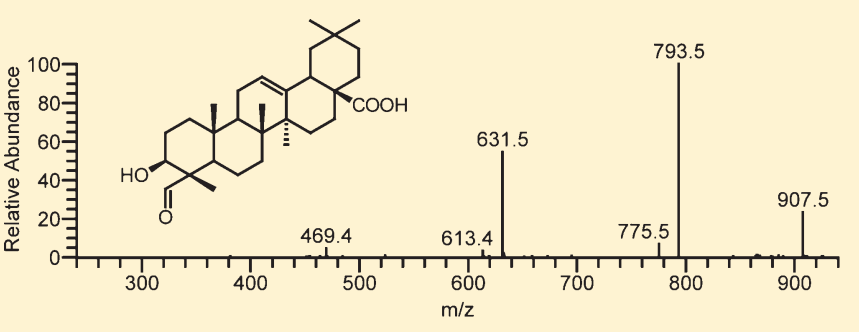
with the generated $\mathrm{MS}^{n}$ spectra, allowed the tentative identification of 79 different saponins, of which 61 had not been detected previously in M. truncatula. Upon collision-induced dissociation of saponins that contain a uronic acid residue in the sugar chain, fragment ions resulting from cross-ring cleavages of the uronic acid residues were observed. The identified saponins are glycosides of 10 different sapogenins, of which three were not detected before in $M$. truncatula. Zanhic acid glycosides, which are prevalent in the aerial parts of $M$. truncatula, were absent in the hairy root extracts. This metabolite compendium will facilitate future functional genomic studies of triterpene saponin biosynthesis in M. truncatula.
\end{abstract}

T egumes (Fabaceae) are important agricultural crops, charLacterized by the presence of root nodules that contain symbiotic nitrogen-fixing bacteria. Fixation of atmospheric nitrogen allows reduced fertilizer costs and makes legumes a key player in crop rotation to replenish nitrogen-depleted soil. Furthermore, legumes serve as a major source of proteins and oil for human and animal nutrition. Leguminous crops with a significant economical value include oilseed crops such as soybean (Glycine max) and peanut (Arachis hypogaea), forage and soil-conditioning crops such as alfalfa (Medicago sativa) and clover (Trifolium spp.), and pulses such as beans (Phaseolus spp.), peas (Pisum spp.), and lentils (Lens spp.).

The more than 19400 leguminous species, distributed over 730 genera, produce a vast array of metabolites with diverse structures and biological activities. An important group of legume natural products are triterpene saponins, a class of secondary metabolites that display a wide range of biological activities. ${ }^{1}$ In plants they serve as allelopathic, antipalatability, anti-insect, or antifungal agents. ${ }^{2}$ Saponins also have a broad range of pharmaceutical properties, such as anti-inflammatory, antimicrobial, anticancer, or adjuvant activities. ${ }^{3,4}$ Besides saponins, isoflavonoids, alkaloids, and nonprotein amino acids commonly occur as natural products in several legume species. ${ }^{1,5}$

As a model species for legume biology, barrel medic (Medicago truncatula), a close relative of alfalfa, is an excellent system to study secondary metabolism of legumes. ${ }^{1,6}$ Consequently, several studies about the metabolite composition of M. truncatula have been published over the past decade. Metabolite profiling using LC-MS-based methods revealed the presence of a complex mixture of triterpene saponins in various $M$. truncatula tissues. ${ }^{2,7-9}$ In accordance with other legumes from the Papilionoideae subfamily, M. truncatula is rich in isoflavonoids. ${ }^{10,11}$

Hairy roots are generated by an infection of healthy plant tissue, not necessarily roots, with Agrobacterium rhizogenes. They are characterized by fast growth rates and do not require growth regulators for their cultivation. Furthermore, they have a high genetic stability and a high biosynthetic potential for a longer time than natural roots. ${ }^{12,13}$ Their potential to produce compounds that are also naturally occurring in wild-type roots in combination with their tolerance to cultivation in large-scale bioreactors makes hairy roots an appealing alternative for the destructive harvesting of wild-type roots, especially those of endangered plant species, to obtain valuable phytochemicals. ${ }^{14,15}$ Hairy roots are also a valuable tool to investigate the secondary metabolism in M. truncatula. A prerequisite for the study of secondary metabolism and its application in metabolic engineering projects is the possibility to generate transgenic plant tissue. Although fertile transgenic $M$. truncatula plants can be obtained via Agrobacterium tumefaciens-mediated transformation, ${ }^{16-19}$ the

Received: $\quad$ March 9, 2011

Published: May 26, 2011 
A ctr1b RT: 23.19 AV: 1 NL: 5.33E4

T: FTMS - p ESI Full ms [120.00-1400.00]

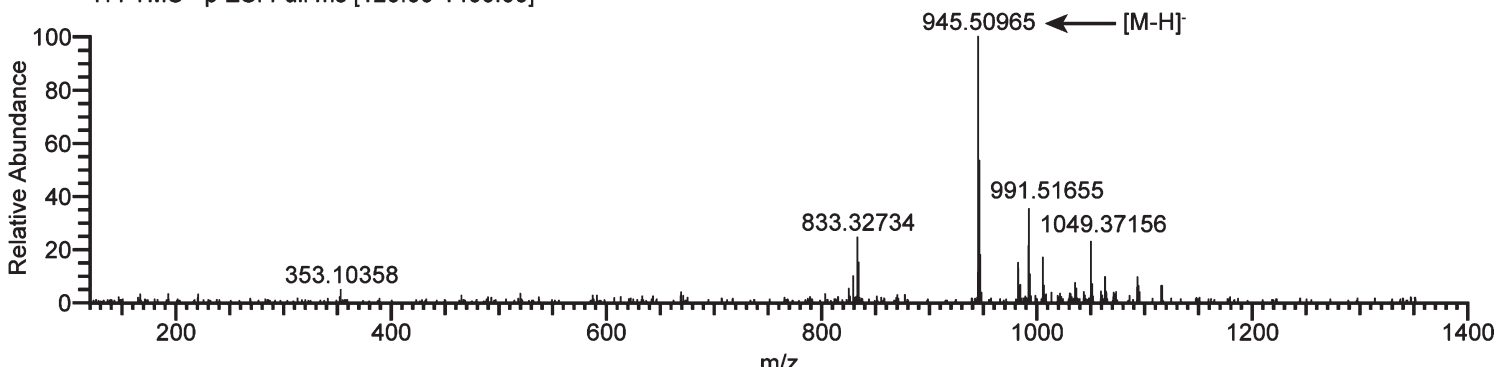

B ctr1b RT: 23.19 AV: 1 NL: 5.33E4

T: FTMS - p ESI Full ms [120.00-1400.00]

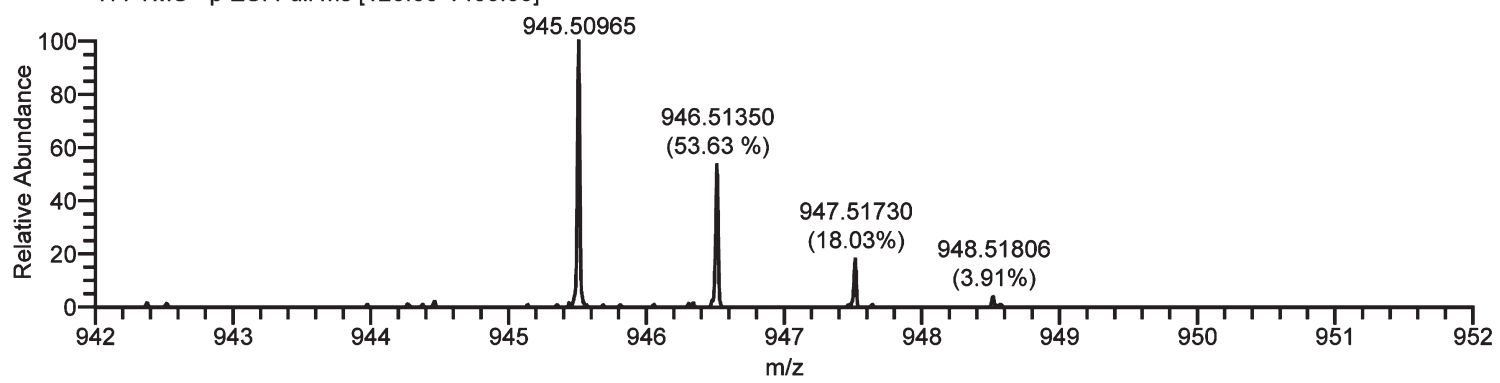

C ctr1b RT: 23.20 AV: 1 NL: 3.32E3

T: ITMS - c ESI d Full ms2945.51@cid35.00 [250.00-960.00]

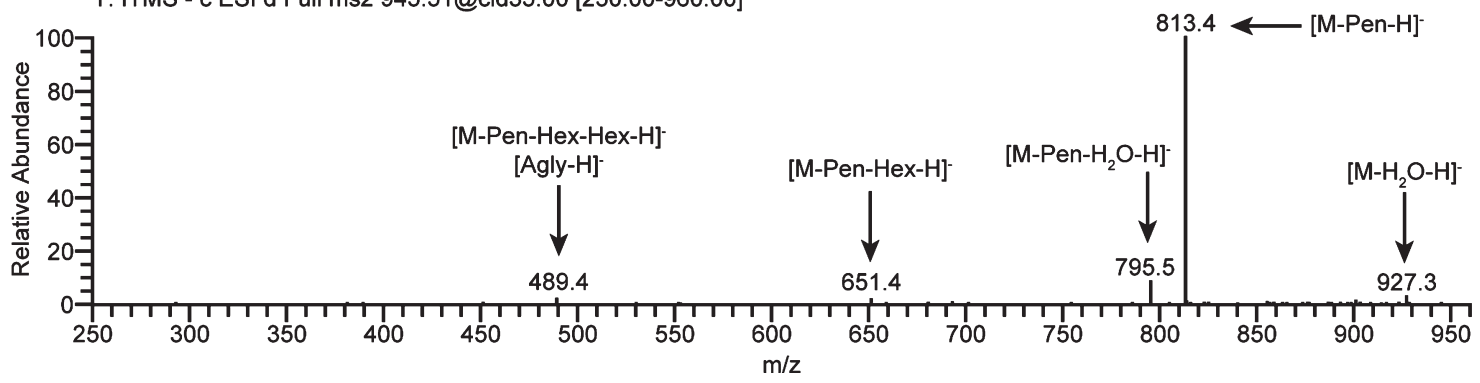

Figure 1. LC ESI FT-ICR MS chromatograms. (A) MS scan of peak at $t_{\mathrm{R}} 23.19 \mathrm{~min}$. (B) Zoom of the MS scan of panel A, showing that the quasimolecular ion is accompanied by additional isotope ions. The relative intensity of the isotope ions is indicated in brackets. (C) MS ${ }^{2}$ fragmentation of the $[\mathrm{M}-\mathrm{H}]^{-}$ion at $m / z 945.51$ (Pen-Hex-Hex-aglycone D).

published methods are time-consuming and lack the required throughput for simultaneous analysis of a significant number of genes. In contrast, $A$. rhizogenes-mediated transformation of $M$. truncatula seedlings allows a fast and efficient production of transgenic hairy roots; ${ }^{20}$ the generation time needed from seed to a transgenic $M$. truncatula hairy root culture, ready for profiling or other analysis, is only three months.

For any metabolic engineering project targeting triterpene saponin biosynthesis, a detailed analysis of the saponin composition of the generated hairy roots is required. In the past decade, a few metabolic profiling studies on saponins in M. truncatula have been reported. In a first study, the presence of a large number of saponins in root extracts of $M$. truncatula was shown: based on fragmentation data under negative ionization, $27 \mathrm{M}$. truncatula saponins were tentatively identified. ${ }^{2}$ Optimization of the latter method resulted in an extended list of 31 tentatively identified saponins. ${ }^{7}$ In a later study, 12 additional saponins from the aerial parts of $M$. truncatula were characterized via spectroscopic methods, and their fragmentation behavior under negative ionization was reported. ${ }^{8,9}$ Nevertheless, $M$. truncatula was postulated to synthesize many more triterpene saponins than identified to date. ${ }^{2}$

For identification purposes, LC-MS-based metabolomics studies benefit from the use of Fourier transform ion cyclotron resonance mass spectrometry (FT-ICR MS). FT-ICR MS provides highly accurate mass measurements, allowing a reliable prediction of the molecular formula of the detected ions. Here, we applied reversed-phase liquid chromatography (LC) coupled to negative-ion electrospray ionization (ESI) FT-ICR MS to investigate the saponin composition of hairy roots of $M$. truncatula. As a result, 79 saponins were detected and tentatively identified, of which soyasaponin I and 3-Glc-28-Glc-medicagenic acid were the most abundant compounds. The identified saponins are glycosides of 10 different sapogenins, of which three had hitherto not been detected before in M. truncatula.

\section{RESULTS AND DISCUSSION}

General Methodology. For metabolite profiling of triterpene saponins, the compounds from a methanol extract of $M$. truncatula hairy roots were separated by reversed-phase LC, followed by negative-ion ESI FT-ICR MS. The accurate $m / z$ measurements of the FT-ICR MS, combined with the isotope abundances, allowed us to predict the molecular formula of the detected saponins. The structural information needed for the identification of the saponins was obtained by $\mathrm{MS}^{n}$ fragmentation using the linear ion trap (IT). Full FT-MS spectra were 
Table 1. Observed Saponins from M. truncatula Hairy Roots, Tentative Identification, and FT-ICR MS ${ }^{n}$ Characteristics $^{a}$

\begin{tabular}{|c|c|c|c|c|}
\hline & $\begin{array}{l}\text { Peak numbers and tentative } \\
\text { dentification of the observed saponins }\end{array}$ & s $[\mathrm{M}-\mathrm{H}]^{-}$ & $\begin{array}{l}\text { Formula } \\
(\delta \text { ppm })\end{array}$ & FT-ICR MS ${ }^{n}: m / z$ (\% base peak) \\
\hline 1 & dHex-Hex-HexA-Aglycone D & 973.50386 & $\mathrm{C}_{48} \mathrm{H}_{78} \mathrm{O}_{20}(2.560)$ & 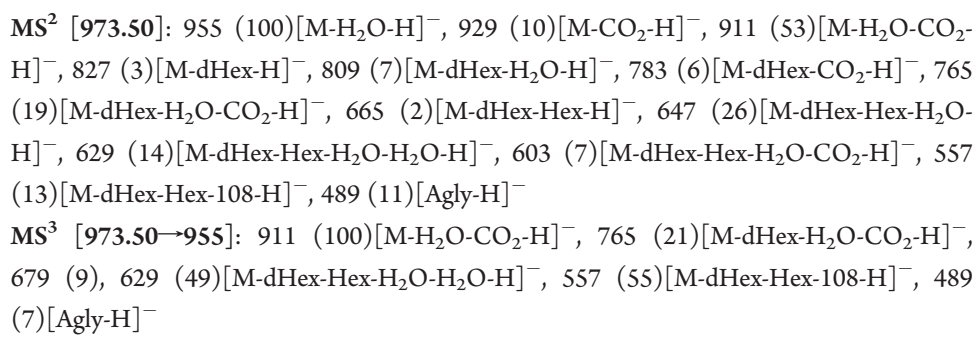 \\
\hline 2 & Pen-Hex-Hex-Aglycone D & 945.50965 & $\mathrm{C}_{47} \mathrm{H}_{78} \mathrm{O}_{19}(3.380)$ & 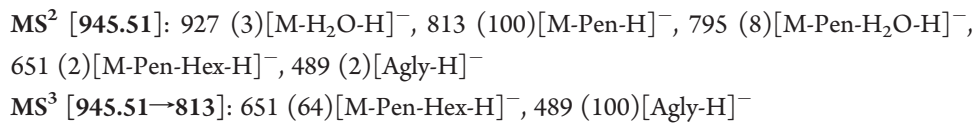 \\
\hline 3 & Hex-Hex-HexA-Bayogenin & 987.48370 & $\mathrm{C}_{48} \mathrm{H}_{76} \mathrm{O}_{21}(3.106)$ & $\begin{array}{l}\mathbf{M S}^{2}[\mathbf{9 8 7 . 4 8}]: 825(4)[\mathrm{M}-\mathrm{Hex}-\mathrm{H}]^{-}, 807(100)\left[\mathrm{M}-\mathrm{Hex}-\mathrm{H}_{2} \mathrm{O}-\mathrm{H}\right]^{-}, 645(10)[\mathrm{M}-\mathrm{Hex}-\mathrm{Hex}- \\
\left.\mathrm{H}_{2} \mathrm{O}-\mathrm{H}\right]^{-}, 601(18)\left[\mathrm{M}-\mathrm{Hex}-\mathrm{Hex}-\mathrm{H}_{2} \mathrm{O}-\mathrm{CO}_{2}-\mathrm{H}\right]^{-}, 487(2)[\text { Agly-H}]^{-} \\
\mathbf{M S}^{3}[\mathbf{9 8 7 . 4 8} \rightarrow \mathbf{8 0 7}]: 645(85)\left[\mathrm{M}-\mathrm{Hex}-\mathrm{Hex}-\mathrm{H}_{2} \mathrm{O}-\mathrm{H}\right]^{-}, 601(100)\left[\mathrm{M}-\mathrm{Hex}-\mathrm{Hex}-\mathrm{H}_{2} \mathrm{O}-\mathrm{CO}_{2}-\right. \\
\mathrm{H}^{-}, 487(12)[\text { Agly-H}]^{-}\end{array}$ \\
\hline 4 & $\begin{array}{l}\text { Hex-dHex-Hex-Hex-Bayogenin } \\
\text { dHex-Hex-Hex-Bayogenin }{ }^{b}\end{array}$ & $\begin{array}{l}1119.56348 \\
957.51011^{b}\end{array}$ & $\begin{array}{l}\mathrm{C}_{54} \mathrm{H}_{88} \mathrm{O}_{24}(3.754) \\
\mathrm{C}_{48} \mathrm{H}_{78} \mathrm{O}_{19}(3.818)\end{array}$ & $\begin{array}{l}\text { MS }^{2}[1119.56]: 957(100)[\text { M-Hex-H }]^{-}, 811(54)[\text { M-Hex-dHex-H }]^{-}, 793(9)[\text { M-Hex- } \\
\left.\text { dHex- }{ }_{2} \mathrm{O}-\mathrm{H}\right]^{-}, 649(33)[\text { M-Hex-dHex-Hex-H }]^{-}, 631(13)\left[\text { M-Hex-dHex-Hex-H }{ }_{2} \mathrm{O}-\mathrm{H}\right]^{-}, \\
487(9)[\text { Agly-H}]^{-} \\
\text {MS }^{3}[1119.56 \rightarrow 957]: 811(100)[\text { M-Hex-dHex-H }]^{-}, 793(6)\left[\text { M-Hex-dHex-H }{ }_{2} \mathrm{O}-\mathrm{H}\right]^{-}, 649 \\
(11)[\text { M-Hex-dHex-Hex-H }]^{-}, 631(3)\left[\text { M-Hex-dHex-Hex-H }{ }_{2} \mathrm{O}-\mathrm{H}\right]^{-}, 487(2)[\text { Agly-H }]^{-} \\
\text {MS }^{3}[1119.56 \rightarrow 811]: 649(100)[\text { M-Hex-dHex-Hex-H }]^{-}, 631(8)\left[\text { M-Hex-dHex-Hex-H }{ }_{2} \mathrm{O}-\right. \\
\mathrm{H}^{-}, 487(68)[\text { Agly-H }]^{-}\end{array}$ \\
\hline 5 & $\begin{array}{l}\text { Malonyl-Hex-Hex-HexA- } \\
\text { Bayogenin }\end{array}$ & 1073.48680 & $\mathrm{C}_{51} \mathrm{H}_{78} \mathrm{O}_{24}(5.378)$ & 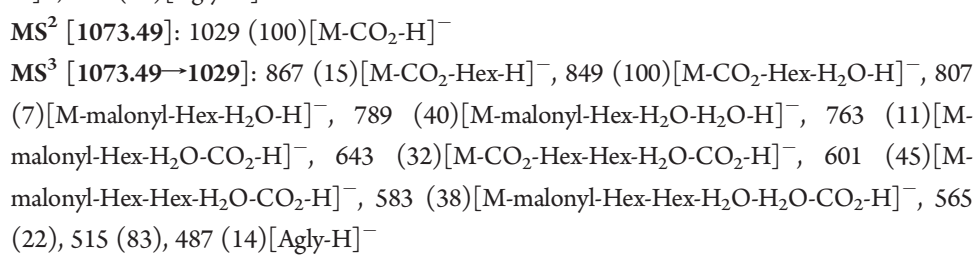 \\
\hline 6 & Hex-Hex-Hex-Bayogenin & 973.50421 & $\mathrm{C}_{48} \mathrm{H}_{78} \mathrm{O}_{20}$ (2.919) & $\begin{array}{l}\text { MS }^{2}[973.50]: 955(10)\left[\mathrm{M}-\mathrm{H}_{2} \mathrm{O}-\mathrm{H}\right]^{-}, 811(100)[\mathrm{M}-\mathrm{Hex}-\mathrm{H}]^{-}, 793(2)\left[\mathrm{M}-\mathrm{Hex}-\mathrm{H}_{2} \mathrm{O}-\mathrm{H}\right]^{-}, \\
649(20)[\mathrm{M}-\mathrm{Hex}-\mathrm{Hex}-\mathrm{H}]^{-}, 631(7)\left[\mathrm{M}-\mathrm{Hex}-\mathrm{Hex}-\mathrm{H}_{2} \mathrm{O}-\mathrm{H}\right]^{-}, 487(8)[\text { Agly-H}]^{-} \\
\text {MS }^{3}[\mathbf{9 7 3 . 5 0 \rightarrow 8 1 1}]: 649(100)[\mathrm{M}-\mathrm{Hex}-\mathrm{Hex}-\mathrm{H}]^{-}, 631(20)\left[\mathrm{M}-\mathrm{Hex}-\mathrm{Hex}_{-} \mathrm{H}_{2} \mathrm{O}-\mathrm{H}\right]^{-}, 487 \\
(36)[\text { Agly-H}]^{-} \\
\text {MS }^{3}\left[\mathbf{9 7 3 . 5 0 \rightarrow 6 4 9 ] :} 487(100)[\text { Agly-H}]^{-}\right.\end{array}$ \\
\hline 7 & $\begin{array}{l}\text { dHex-Hex-HexA- } \\
\text { Soyasapogenol A }\end{array}$ & 957.50926 & $\mathrm{C}_{48} \mathrm{H}_{78} \mathrm{O}_{19}(2.931)$ & $\begin{array}{l}\mathrm{MS}^{2}[957.51]: 939(100)\left[\mathrm{M}-\mathrm{H}_{2} \mathrm{O}-\mathrm{H}\right]^{-}, 895(49)\left[\mathrm{M}-\mathrm{H}_{2} \mathrm{O}-\mathrm{CO}_{2}-\mathrm{H}\right]^{-}, 811(5)[\mathrm{M}-\mathrm{dHex}-\mathrm{H}]^{-}, \\
767(3)\left[\mathrm{M}-\mathrm{dHex}-\mathrm{CO}_{2}-\mathrm{H}\right]^{-}, 749(21)\left[\mathrm{M}-\mathrm{dHex}-\mathrm{H}_{2} \mathrm{O}-\mathrm{CO}_{2}-\mathrm{H}\right]^{-}, 631(25)[\mathrm{M}-\mathrm{dHex}-\mathrm{Hex}- \\
\left.\mathrm{H}_{2} \mathrm{O}-\mathrm{H}\right]^{-}, 613 \quad(18)\left[\mathrm{M}-\mathrm{dHex}-\mathrm{Hex}-\mathrm{H}_{2} \mathrm{O}-\mathrm{H}_{2} \mathrm{O}-\mathrm{H}\right]^{-}, \quad 541 \quad(18)[\mathrm{M}-\mathrm{dHex}-\mathrm{Hex}-108-\mathrm{H}]^{-}, \\
473(11)[\mathrm{Agly}-\mathrm{H}]^{-}, 453(5) \\
\mathrm{MS}^{3}[957.51 \rightarrow 939]: 895(83)\left[\mathrm{M}-\mathrm{H}_{2} \mathrm{O}-\mathrm{CO}_{2}-\mathrm{H}\right]^{-}, 749(19)\left[\mathrm{M}-\mathrm{dHex}-\mathrm{H}_{2} \mathrm{O}-\mathrm{CO}_{2}-\mathrm{H}\right]^{-}, 613 \\
(61)\left[\mathrm{M}-\mathrm{dHex}-\mathrm{Hex}-\mathrm{H}_{2} \mathrm{O}-\mathrm{H}_{2} \mathrm{O}-\mathrm{H}\right]^{-}, 541(100)[\mathrm{M}-\mathrm{dHex}-\mathrm{Hex}-108-\mathrm{H}]^{-}, 473(4)[\text { Agly-H }]^{-}, \\
453(2)\end{array}$ \\
\hline 8 & Hex-Hex-HexA-Aglycone A & 985.46884 & $\mathrm{C}_{48} \mathrm{H}_{74} \mathrm{O}_{21}(3.914)$ & 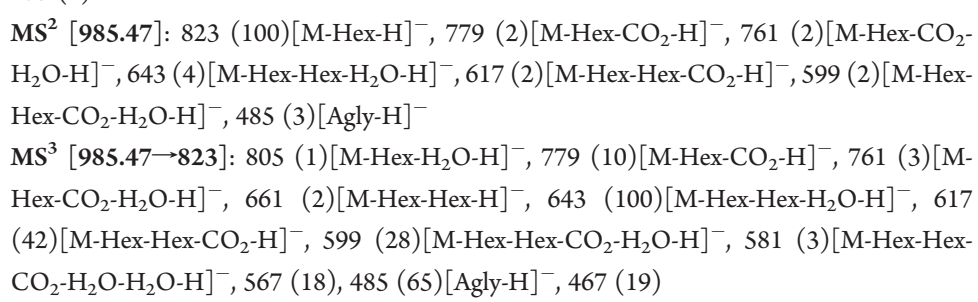 \\
\hline & Hex-Hex-Hex-Bayogenin & 973.50407 & $\mathrm{C}_{48} \mathrm{H}_{78} \mathrm{O}_{20}(2.776)$ & $\begin{array}{l}\text { MS }^{2}[973.50]: 955(10)\left[\mathrm{M}-\mathrm{H}_{2} \mathrm{O}-\mathrm{H}\right]^{-}, 811(100)[\mathrm{M}-\mathrm{Hex}-\mathrm{H}]^{-}, 649(20)[\mathrm{M}-\mathrm{Hex}-\mathrm{Hex}-\mathrm{H}]^{-}, \\
631(7)\left[\mathrm{M}-\mathrm{Hex}-\mathrm{Hex}-\mathrm{H}_{2} \mathrm{O}-\mathrm{H}\right]^{-}, 487(8)[\text { Agly-H}]^{-} \\
\mathbf{M S}^{3}[\mathbf{9 7 3 . 5 0 \rightarrow 8 1 1}]: 649(100)[\mathrm{M}-\mathrm{Hex}-\mathrm{Hex}-\mathrm{H}]^{-}, 631(42)\left[\mathrm{M}-\mathrm{Hex}-\mathrm{Hex}-\mathrm{H}_{2} \mathrm{O}-\mathrm{H}\right]^{-}, 487 \\
(86)[\text { Agly-H}]^{-} \\
\mathbf{M S}^{3}[\mathbf{9 7 3 . 5 0 \rightarrow 6 4 9}]: 487(100)[\text { Agly-H}]^{-} 409(1), 393(1), 391(1)\end{array}$ \\
\hline
\end{tabular}


Table 1. Continued

\begin{tabular}{|c|c|c|c|}
\hline $\begin{array}{l}\text { Peak numbers and tentative } \\
\text { identification of the observed saponin }\end{array}$ & s $[\mathrm{M}-\mathrm{H}]^{-}$ & $\begin{array}{l}\text { Formula } \\
(\delta \mathrm{ppm})\end{array}$ & FT-ICR MS ${ }^{n}: m / z$ (\% base peak) \\
\hline 10 Hex-Hex-Aglycone C & 823.41585 & $\mathrm{C}_{42} \mathrm{H}_{64} \mathrm{O}_{16}(4.481)$ & $\begin{array}{l}\mathrm{MS}^{2}[\mathbf{8 2 3 . 4 2}]: 805(4)\left[\mathrm{M}-\mathrm{H}_{2} \mathrm{O}-\mathrm{H}\right]^{-}, 661(2)[\mathrm{M}-\mathrm{Hex}-\mathrm{H}]^{-}, 617(3)\left[\mathrm{M}-\mathrm{Hex}-\mathrm{CO}_{2}-\mathrm{H}\right]^{-}, 599 \\
(7)\left[\mathrm{M}-\mathrm{Hex}-\mathrm{CO}_{2}-\mathrm{H}_{2} \mathrm{O}-\mathrm{H}\right]^{-}, 437(100)\left[\text { Agly- } \mathrm{CO}_{2}-\mathrm{H}_{2} \mathrm{O}-\mathrm{H}\right]^{-} \\
\mathbf{M S}^{3}[\mathbf{8 2 3 . 4 2 \rightarrow 4 3 7 ]}: 391(100), 375(6)\end{array}$ \\
\hline 11 Hex-HexA-Bayogenin & 825.43079 & $\mathrm{C}_{42} \mathrm{H}_{66} \mathrm{O}_{16}(3.610)$ & $\begin{array}{l}\mathrm{MS}^{2}[825.43]: 807(10)\left[\mathrm{M}-\mathrm{H}_{2} \mathrm{O}-\mathrm{H}\right]^{-}, 781(3)\left[\mathrm{M}-\mathrm{CO}_{2}-\mathrm{H}\right]^{-}, 765(3), 763(2)\left[\mathrm{M}-\mathrm{H}_{2} \mathrm{O}-\mathrm{CO}_{2}-\right. \\
\mathrm{H}^{-}, 705(7), 663(100)[\mathrm{M}-\mathrm{Hex}-\mathrm{H}]^{-}, 645(10)\left[\mathrm{M}-\mathrm{Hex}-\mathrm{H}_{2} \mathrm{O}-\mathrm{H}\right]^{-}, 619(41)\left[\mathrm{M}-\mathrm{Hex}_{-} \mathrm{CO}_{2}\right. \\
\mathrm{H}^{-}, 601(41)\left[\mathrm{M}-\mathrm{Hex}-\mathrm{CO}_{2}-\mathrm{H}_{2} \mathrm{O}-\mathrm{H}\right]^{-}, 587(6)\left[\mathrm{M}-\mathrm{Hex}-\mathrm{CO}_{2}-\mathrm{H}_{2} \mathrm{O}-\mathrm{H}_{2} \mathrm{O}-\mathrm{H}\right]^{-}, 529(6), 487 \\
(34)[\text { Agly-H}]^{-} \\
\mathrm{MS}^{3}[825.43 \rightarrow 663]: 587(4)\left[\mathrm{M}-\mathrm{Hex}-\mathrm{CO}_{2}-\mathrm{H}_{2} \mathrm{O}-\mathrm{H}_{2} \mathrm{O}-\mathrm{H}\right]^{-}, 487(100)[\mathrm{Agly}-\mathrm{H}]^{-}, 467(5), \\
455(6), 439(5)\end{array}$ \\
\hline 12 Hex-Hex-HexA-Hederagenin & 971.48934 & $\mathrm{C}_{48} \mathrm{H}_{76} \mathrm{O}_{20}(3.728)$ & 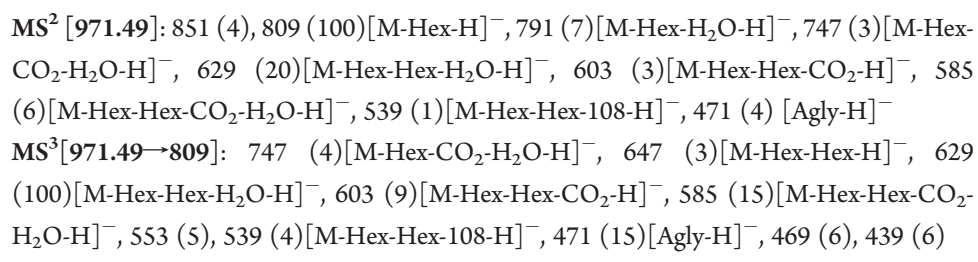 \\
\hline 13 Hex-Hex-Hex-Medicagenic aci & d987.48516 & $\mathrm{C}_{48} \mathrm{H}_{76} \mathrm{O}_{21}$ & $\begin{array}{l}\text { MS }^{2}[\mathbf{9 8 7 . 4 9}]: 825(100)[\mathrm{M}-\mathrm{Hex}-\mathrm{H}]^{-}, 781(5)\left[\mathrm{M}-\mathrm{Hex}-\mathrm{CO}_{2}-\mathrm{H}\right]^{-}, 711(3), 663(4)[\mathrm{M}-\mathrm{Hex}- \\
\mathrm{Hex}-\mathrm{H}]^{-}, 601(4)\left[\mathrm{M}-\mathrm{Hex}-\mathrm{Hex}-\mathrm{CO}_{2}-\mathrm{H}_{2} \mathrm{O}-\mathrm{H}\right]^{-}, 439(53)\left[\text { Agly- } \mathrm{CO}_{2}-\mathrm{H}_{2} \mathrm{O}-\mathrm{H}\right]^{-} \\
\text {MS }^{3}[\mathbf{9 8 7 . 4 9 \rightarrow 8 2 5}]: 439(100)\left[\text { Agly-CO } \mathrm{CO}_{2}-\mathrm{H}_{2} \mathrm{O}-\mathrm{H}\right]^{-}\end{array}$ \\
\hline $\begin{array}{l}14 \text { Malonyl-Hex-Hex-HexA- } \\
\text { Hederagenin }\end{array}$ & $\begin{array}{l}1057.49199 \\
1013.50072^{b}\end{array}$ & $\mathrm{C}_{51} \mathrm{H}_{78} \mathrm{O}_{23}(5.558)$ & 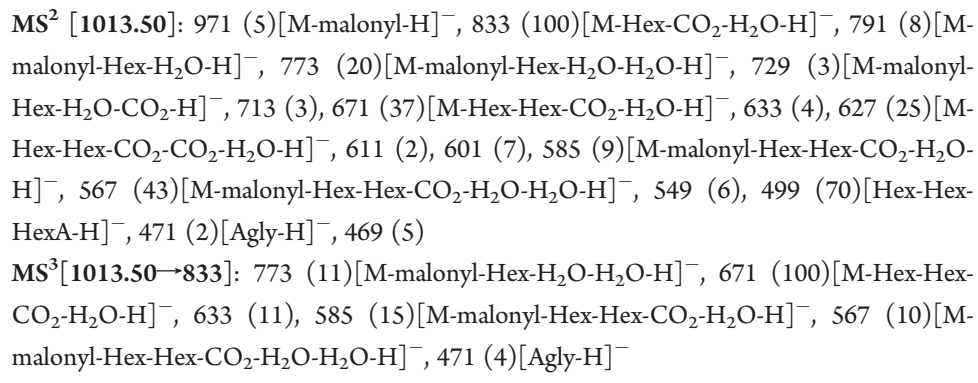 \\
\hline 15 dHex-Hex-Hex-Hederagenin & 941.51456 & $\mathrm{C}_{48} \mathrm{H}_{78} \mathrm{O}_{18}$ (3.209) & $\begin{array}{l}\text { MS }^{2}[\mathbf{9 4 1 . 5 1}]: 795(100)[\mathrm{M}-\mathrm{dHex}-\mathrm{H}]^{-}, 777(10)\left[\mathrm{M}-\mathrm{dHex}-\mathrm{H}_{2} \mathrm{O}-\mathrm{H}\right]^{-}, 633(12)[\mathrm{M}-\mathrm{dHex}- \\
\mathrm{Hex}-\mathrm{H}]^{-}, 615(2)\left[\mathrm{M}-\mathrm{dHex}-\mathrm{Hex}-\mathrm{H}_{2} \mathrm{O}-\mathrm{H}\right]^{-}, 471(1)[\text { Agly-H}]^{-} \\
\text {MS }^{3}[\mathbf{9 4 1 . 5 1 \rightarrow 7 9 5}]: 633(100)[\mathrm{M}-\mathrm{dHex}-\mathrm{Hex}-\mathrm{H}]^{-}, 615(6)\left[\mathrm{M}-\mathrm{dHex}-\mathrm{Hex}-\mathrm{H}_{2} \mathrm{O}-\mathrm{H}\right]^{-}, 471 \\
(25)[\text { Agly-H}]^{-} \\
\text {MS }^{3}\left[\mathbf{9 4 1 . 5 1 \rightarrow 6 3 3 ] : ~} 471(100)[\text { Agly-H}]^{-}, 405(1), 393(1)\right.\end{array}$ \\
\hline 16 Malonyl-Hex-HexA-Bayogenin & $\begin{array}{l}911.43120 \\
867.44138^{b}\end{array}$ & $\mathrm{C}_{45} \mathrm{H}_{68} \mathrm{O}_{19}(3.288)$ & 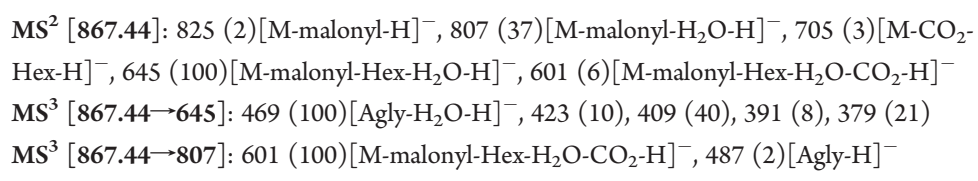 \\
\hline 17 Hex-Hex-HexA-Aglycone A & 985.46931 & $\mathrm{C}_{48} \mathrm{H}_{74} \mathrm{O}_{21}(4.391)$ & $\begin{array}{l}\mathrm{MS}^{2}[\mathbf{9 8 5 . 4 7}]: 823(6)[\mathrm{M}-\mathrm{Hex}-\mathrm{H}]^{-}, 805(100)\left[\mathrm{M}-\mathrm{Hex}-\mathrm{H}_{2} \mathrm{O}-\mathrm{H}\right]^{-}, 643(10)[\mathrm{M}-\mathrm{Hex}-\mathrm{Hex}- \\
\left.\mathrm{H}_{2} \mathrm{O}-\mathrm{H}\right]^{-}, 599(33)\left[\mathrm{M}-\mathrm{Hex}-\mathrm{Hex}-\mathrm{H}_{2} \mathrm{O}-\mathrm{CO}_{2}-\mathrm{H}\right]^{-} \\
\mathbf{M S}^{3}[\mathbf{9 8 5 . 4 7 \rightarrow 8 0 5}]: 685(4), 643(45)\left[\mathrm{M}-\mathrm{Hex}-\mathrm{Hex}-\mathrm{H}_{2} \mathrm{O}-\mathrm{H}\right]^{-}, 599(100)[\mathrm{M}-\mathrm{Hex}-\mathrm{Hex}- \\
\left.\mathrm{H}_{2} \mathrm{O}-\mathrm{CO}_{2}-\mathrm{H}\right]^{-} \\
\mathbf{M S}^{3}[\mathbf{9 8 5 . 4 7 \rightarrow 5 9 9}]: 527(100), 485(2)[\text { Agly-H}]^{-}\end{array}$ \\
\hline 18 Hex-Hex-Bayogenin & 811.45241 & $\mathrm{C}_{42} \mathrm{H}_{68} \mathrm{O}_{15}(4.763)$ & $\begin{array}{l}\text { MS }^{2}[\mathbf{8 1 1 . 4 5}]: 649(100)[\mathrm{M}-\mathrm{Hex}-\mathrm{Hex}-\mathrm{H}]^{-}, 631(8)\left[\mathrm{M}-\mathrm{Hex}-\mathrm{Hex}-\mathrm{H}_{2} \mathrm{O}-\mathrm{H}\right]^{-}, 487(62)[\text { Agly- } \\
\mathrm{H}^{-} \\
\mathbf{M S}^{3}[\mathbf{8 1 1 . 4 5 \rightarrow 6 4 9}]: 487(100)[\text { Agly-H}]^{-} \\
\mathbf{M S}^{3}[\mathbf{8 1 1 . 4 5 \rightarrow 4 8 7}]: 409(100), 403(21), 393(38), 391(74)\end{array}$ \\
\hline 19 Hex-Hex-HexA-Hederagenin & 971.48997 & $\mathrm{C}_{48} \mathrm{H}_{76} \mathrm{O}_{20}$ & 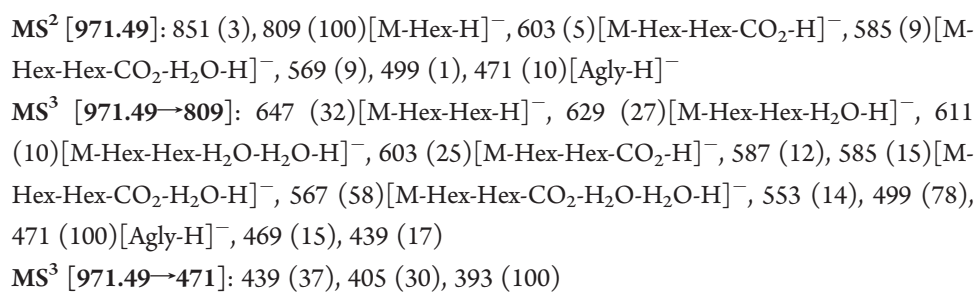 \\
\hline
\end{tabular}


Table 1. Continued

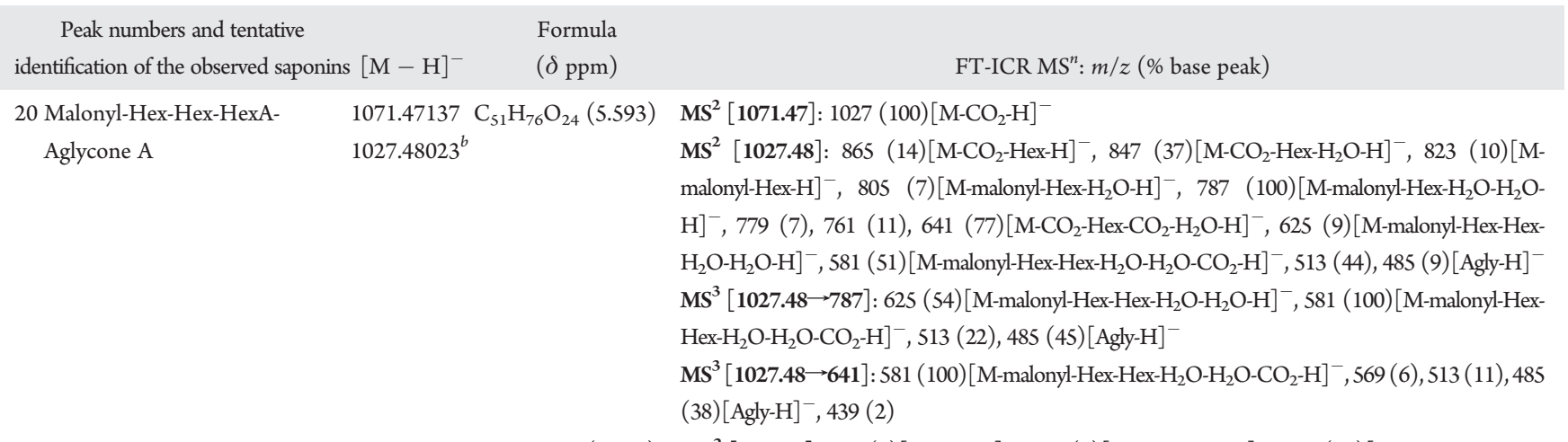

21 3-Glc-28-Glc-Medicagenic acid 825.42987 $\mathrm{C}_{42} \mathrm{H}_{66} \mathrm{O}_{16}(2.494) \quad \mathrm{MS}^{2}[825.43]: 663(5)[\mathrm{M}-\mathrm{Glc}-\mathrm{H}]^{-}, 619(3)\left[\mathrm{M}-\mathrm{Glc}_{-} \mathrm{CO}_{2}-\mathrm{H}\right]^{-}, 601(35)\left[\mathrm{M}-\mathrm{Glc}_{-} \mathrm{CO}_{2}-\mathrm{H}_{2} \mathrm{O}-\right.$ $\mathrm{H}^{-}, 439(100)\left[\text { Agly- } \mathrm{CO}_{2}-\mathrm{H}_{2} \mathrm{O}-\mathrm{H}\right]^{-}$

MS $^{3}$ [825.43 $\left.\rightarrow 439\right]: 393(51), 391(100), 375(8)$

$\mathbf{M S}^{3}[825.43 \rightarrow 601]: 439(100)\left[\text { Agly- } \mathrm{CO}_{2}-\mathrm{H}_{2} \mathrm{O}-\mathrm{H}\right]^{-}$

22 Malonyl-Hex-HexA-Bayogenin $911.43371 \quad \mathrm{C}_{45} \mathrm{H}_{68} \mathrm{O}_{19}(6.041)$ $867.44133^{b}$

$\mathrm{MS}^{2}$ [867.44]: 825 (34)[M-malonyl- $]^{-}, 807$ (25)[M-malonyl- $\left.\mathrm{H}_{2} \mathrm{O}-\mathrm{H}\right]^{-}, 705$ (9) $\left[{\mathrm{M}-\mathrm{CO}_{2}}^{-}\right.$ $\mathrm{Hex}-\mathrm{H}]^{-}, 645$ (77) [M-malonyl-Hex- $\left.\mathrm{H}_{2} \mathrm{O}-\mathrm{H}\right]^{-}, 601(100)\left[\mathrm{M}-\text { malonyl-Hex- } \mathrm{H}_{2} \mathrm{O}-\mathrm{CO}_{2}-\mathrm{H}\right]^{-}$ $\mathbf{M S}^{3}$ [867.44 $\left.\rightarrow 601\right]: 583(3), 529(100), 487(7)[\text { Agly-H }]^{-}$

23 dHex-Hex-Hex-Hederagenin $\quad 941.51536 \quad \mathrm{C}_{48} \mathrm{H}_{78} \mathrm{O}_{18}(4.058)$

MS $^{2}$ [941.51]: 795 (100)[M-dHex-H] $]^{-}, 777$ (12)[M-dHex- $\left.\mathrm{H}_{2} \mathrm{O}-\mathrm{H}\right]^{-}, 633$ (13)[M-dHex$\mathrm{Hex}-\mathrm{H}]^{-}, 615$ (2) $\left[\mathrm{M}-\mathrm{dHex}-\mathrm{Hex}-\mathrm{H}_{2} \mathrm{O}-\mathrm{H}\right]^{-}$

MS $^{3}[$ 941.51 $\rightarrow 795]: 633(100)[\mathrm{M}-\mathrm{dHex}-\mathrm{Hex}-\mathrm{H}]^{-}, 615(5)\left[\mathrm{M}-\mathrm{dHex}-\mathrm{Hex}-\mathrm{H}_{2} \mathrm{O}-\mathrm{H}\right]^{-}, 471$ (20) $[\text { Agly-H }]^{-}$

MS $^{3}$ [941.51 $\rightarrow$ 633]: $471(100)[\text { Agly-H }]^{-}, 393(3)$

24 Hex-Hex-HexA-Bayogenin

25 Hex-dHex-Hex-HexAHederagenin

26 3-Glc-Malonyl-28-GlcMedicagenic acid

27 Hex-Bayogenin 28 Hex-Medicagenic acid 29 Hex-HexA-Aglycone A

$\begin{array}{ccc}30 \text { Malonyl-Hex-Hex-Medicagenic } & 911.43152 & \mathrm{C}_{45} \mathrm{H}_{68} \mathrm{O}_{19}(3.638) \\ \text { acid } & 867.44123^{b}\end{array}$ 31 dHex-Hex-HexA-Hederagenin $955.49433 \quad \mathrm{C}_{48} \mathrm{H}_{76} \mathrm{O}_{19}(3.690)$
$\mathrm{MS}^{2}$ [987.48]: 969 (3) [M- $\left.\mathrm{H}_{2} \mathrm{O}-\mathrm{H}\right]^{-}, 825$ (100)[M-Hex-H $]^{-}, 645$ (2)[M-Hex-Hex- $\mathrm{H}_{2} \mathrm{O}-$ $\mathrm{H}]^{-}, 601(2)\left[\mathrm{M}-\mathrm{Hex}-\mathrm{Hex}-\mathrm{H}_{2} \mathrm{O}-\mathrm{CO}_{2}-\mathrm{H}\right]^{-}, 487(3)[\text { Agly-H}]^{-}$

$\mathrm{MS}^{3}[\mathbf{9 8 7 . 4 8 \rightarrow 8 2 5}]: 781(4)\left[\mathrm{M}-\mathrm{Hex}-\mathrm{CO}_{2}-\mathrm{H}\right]^{-}, 645(88)\left[\mathrm{M}-\mathrm{Hex}-\mathrm{Hex}-\mathrm{H}_{2} \mathrm{O}-\mathrm{H}\right]^{-}, 619$ (24) $[\mathrm{M}-\mathrm{Hex}-$ $\left.\mathrm{Hex}-\mathrm{CO}_{2}-\mathrm{H}\right]^{-}, 601$ (29) [M-Hex-Hex- $\left.\mathrm{H}_{2} \mathrm{O}-\mathrm{CO}_{2}-\mathrm{H}\right]^{-}, 569$ (23), 487 (100) $[\mathrm{Agly}-\mathrm{H}]^{-}, 467$ (3)

1117.54979 $\mathrm{C}_{54} \mathrm{H}_{86} \mathrm{O}_{24}(5.515) \quad \mathrm{MS}^{2}[1117.55]: 1099(5)\left[\mathrm{M}-\mathrm{H}_{2} \mathrm{O}-\mathrm{H}\right]^{-}, 955(100)[\mathrm{M}-\mathrm{Hex}-\mathrm{H}]^{-}, 937$ (4) $\left[\mathrm{M}-\mathrm{Hex}-\mathrm{H}_{2} \mathrm{O}-\mathrm{H}\right]^{-}$, 893 (4) $\left[\mathrm{M}-\mathrm{Hex}-\mathrm{H}_{2} \mathrm{O}-\mathrm{CO}_{2}-\mathrm{H}\right]^{-}, 791$ (1) $\left[\mathrm{M}-\mathrm{Hex}-\mathrm{dHex}-\mathrm{H}_{2} \mathrm{O}-\mathrm{H}\right]^{-}, 747$ (1)[M-Hex- dHex$\left.\mathrm{H}_{2} \mathrm{O}-\mathrm{CO}_{2}-\mathrm{H}\right]^{-}, 629$ (2)[M-Hex-dHex-Hex-H] $]^{-}, 539$ (2)[M-Hex-dHex-Hex-108-H] MS $^{2}[1117.55 \rightarrow 955]: 937(88)\left[\mathrm{M}-\mathrm{Hex}-\mathrm{H}_{2} \mathrm{O}-\mathrm{H}\right]^{-}, 893(67)\left[\mathrm{M}-\mathrm{Hex}-\mathrm{H}_{2} \mathrm{O}-\mathrm{CO}_{2}-\mathrm{H}\right]^{-}, 747$ (67) $\left[\mathrm{M}-\mathrm{Hex}-\mathrm{dHex}-\mathrm{H}_{2} \mathrm{O}-\mathrm{CO}_{2}-\mathrm{H}\right]^{-}, \quad 729 \quad(21)\left[\mathrm{M}-\mathrm{Hex}-\mathrm{dHex}-\mathrm{H}_{2} \mathrm{O}-\mathrm{H}_{2} \mathrm{O}-\mathrm{CO}_{2}-\mathrm{H}\right]^{-}, \quad 629$ (100)[M-Hex-dHex-Hex-H] $]^{-}, 611$ (15)[M-Hex-dHex-Hex-H $\left.{ }_{2} \mathrm{O}-\mathrm{H}\right]^{-}, \quad 585$ (14)[M-HexdHex-Hex-CO $\left.\mathrm{CO}_{2}-\mathrm{H}\right]^{-}, 539$ (16)[M-Hex-dHex-Hex-108-H] $]^{-}, 471(17)[\text { Agly-H }]^{-}, 453$ (31)

$911.43233 \mathrm{C}_{45} \mathrm{H}_{68} \mathrm{O}_{19}(4.522) \quad \mathbf{M S}^{2}[911.43]: 867(100)\left[\mathrm{M}-\mathrm{CO}_{2}-\mathrm{H}\right]^{-}, 705(3)\left[\mathrm{M}-\mathrm{Glc}_{-} \mathrm{CO}_{2}-\mathrm{H}\right]^{-}$

$\operatorname{MS}^{3}[911.43 \rightarrow 867]: 705(4)\left[\mathrm{M}-\mathrm{Glc}-\mathrm{CO}_{2}-\mathrm{H}\right]^{-}, 661(7)\left[\mathrm{M}-\mathrm{Glc}_{-} \mathrm{CO}_{2}-\mathrm{CO}_{2}-\mathrm{H}\right]^{-}, 601$ (36) $\left[\mathrm{M}-\text { Glc-malonyl- } \mathrm{CO}_{2}-\mathrm{H}_{2} \mathrm{O}-\mathrm{H}\right]^{-}, 439(100)\left[\text { Agly- } \mathrm{CO}_{2}-\mathrm{H}_{2} \mathrm{O}-\mathrm{H}\right]^{-}$

$649.39774 \mathrm{C}_{36} \mathrm{H}_{58} \mathrm{O}_{10}(3.108) \quad \mathrm{MS}^{2}[649.40]: 487$ (100)[Agly-H] $]^{-}, 469$ (15), 441 (28), 409 (36)

$663.37701 \quad \mathrm{C}_{36} \mathrm{H}_{56} \mathrm{O}_{11}(3.051) \quad \mathrm{MS}^{2}[663.38]: 645(5)\left[\mathrm{M}-\mathrm{H}_{2} \mathrm{O}-\mathrm{H}\right]^{-}, 501(13)[\mathrm{Agly}-\mathrm{H}]^{-}, 439(100)\left[\mathrm{Agly}-\mathrm{CO}_{2}-\mathrm{H}_{2} \mathrm{O}-\mathrm{H}\right]^{-}$

$\mathrm{MS}^{3}[663.38 \rightarrow 439]: 393(83), 391(100)$

$823.41492 \mathrm{C}_{42} \mathrm{H}_{64} \mathrm{O}_{16}(3.352) \quad \mathrm{MS}^{2}[823.41]: 661(100)[\mathrm{M}-\mathrm{Hex}-\mathrm{H}]^{-}, 617(33)\left[\mathrm{M}-\mathrm{Hex}-\mathrm{CO}_{2}-\mathrm{H}\right]^{-}, 599(52)\left[\mathrm{M}-\mathrm{Hex}-\mathrm{H}_{2} \mathrm{O}-\right.$ $\left.\mathrm{CO}_{2}-\mathrm{H}\right]^{-}, 485(15)[\text { Agly-H }]^{-}$

MS $^{3}[823.41 \rightarrow 661]: 585(5), 485(100)[\text { Agly-H }]^{-}, 467(34)$

$\mathbf{M S}^{3}$ [823.41 $\left.\rightarrow 599\right]: 527(100), 485(3)[\text { Agly-H }]^{-}, 467(2)$

$\mathrm{MS}^{2}[867.44]: 825$ (33)[M-malonyl-H $]^{-}, 807$ (16)[M-malonyl- $\left.\mathrm{H}_{2} \mathrm{O}-\mathrm{H}\right]^{-}, 705$ (12)[M-Hex$\left.\mathrm{CO}_{2}-\mathrm{H}\right]^{-}, \quad 663$ (10)[M-malonyl-Hex-H] $]^{-}, \quad 645$ (63)[M-malonyl-Hex-H $\left.\mathrm{H}_{2} \mathrm{O}-\mathrm{H}\right]^{-}, 601$ (100)[M-malonyl-Hex- $\left.\mathrm{H}_{2} \mathrm{O}-\mathrm{CO}_{2}-\mathrm{H}\right]^{-}, 487$ (9), 439 (82) $\left[\text { Agly- } \mathrm{CO}_{2}-\mathrm{H}_{2} \mathrm{O}-\mathrm{H}\right]^{-}$

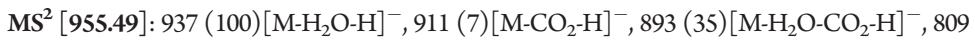
(8) $[\mathrm{M}-\mathrm{dHex}-\mathrm{H}]^{-}, 747$ (24) $\left[\mathrm{M}-\mathrm{dHex}-\mathrm{H}_{2} \mathrm{O}-\mathrm{CO}_{2}-\mathrm{H}\right]^{-}, 629$ (28) $\left[\mathrm{M}-\mathrm{dHex}-\mathrm{Hex}-\mathrm{H}_{2} \mathrm{O}-\mathrm{H}\right]^{-}, 611$ (11) $\left[\mathrm{M}-\mathrm{dHex}-\mathrm{Hex}-\mathrm{H}_{2} \mathrm{O}-\mathrm{H}_{2} \mathrm{O}-\mathrm{H}\right]^{-}, 539$ (22) $[\mathrm{M}-\mathrm{dHex}-\mathrm{Hex}-108-\mathrm{H}]^{-}, 471$ (4) $[\text { Agly-H }]^{-}$ $\mathrm{MS}^{3}[$ [955.49 $\rightarrow 937]: 893(100)\left[\mathrm{M}-\mathrm{H}_{2} \mathrm{O}-\mathrm{CO}_{2}-\mathrm{H}\right]^{-}, 859(6), 747(20)\left[\mathrm{M}-\mathrm{dHex}-\mathrm{H}_{2} \mathrm{O}-\mathrm{CO}_{2}-\mathrm{H}\right]^{-}$, 611 (66) [M-dHex-Hex- $\left.\mathrm{H}_{2} \mathrm{O}-\mathrm{H}_{2} \mathrm{O}-\mathrm{H}\right]^{-}, 539$ (74) $[\mathrm{M}-\mathrm{dHex}-\mathrm{Hex}-108-\mathrm{H}]^{-}$, 471 (13) $[\mathrm{Agly}-\mathrm{H}]^{-}$, 451 (4), 441 (9)

MS $^{3}$ [955.49 $\left.\rightarrow 893\right]: 747(100)\left[\mathrm{M}-\mathrm{dHex}-\mathrm{H}_{2} \mathrm{O}-\mathrm{CO}_{2}-\mathrm{H}\right]^{-}, 729$ (2), 585 (3), 567 (5), 471 (12) $[\text { Agly-H }]^{-}$ 
Table 1. Continued

\begin{tabular}{|c|c|c|}
\hline $\begin{array}{l}\text { Peak numbers and tentative } \\
\text { identification of the observed saponins }[\mathrm{M}-\mathrm{H}]^{-}\end{array}$ & $\begin{array}{l}\text { Formula } \\
(\delta \mathrm{ppm})\end{array}$ & FT-ICR MS ${ }^{n}: m / z$ (\% base peak) \\
\hline $\begin{array}{l}32 \text { Hex-dHex-Hex-HexA- } \\
\text { Soyasapogenol E }\end{array}$ & $\mathrm{C}_{54} \mathrm{H}_{86} \mathrm{O}_{23}(4.673)$ & 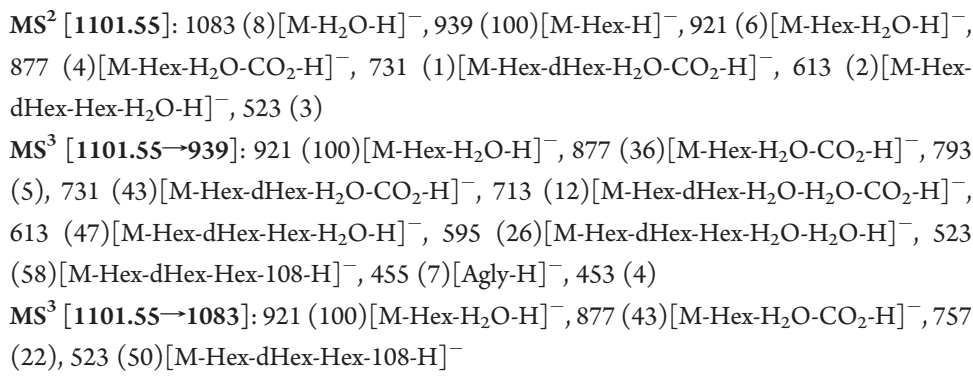 \\
\hline
\end{tabular}

33 Hex-Hex-HexA-Hederagenin

34 dHex-Hex-Hex-HexA-

Soyasapogenol B

35 Malonyl-Hex-Malonyl-HexBayogenin

36 Malonyl-Hex-Malonyl-HexMedicagenic acid

37 dHex-Hex-HexASoyasapogenol A

38 Malonyl-Hex-HexAHederagenin

39 dHex-Hex-HexA-Hederagenin $865.42579^{b} \quad \mathrm{C}_{44} \mathrm{H}_{66} \mathrm{O}_{17}(3.543)$
983.45391 $\quad \mathrm{C}_{48} \mathrm{H}_{72} \mathrm{O}_{21}$ (4.654)

997.4345 $\quad \mathrm{C}_{48} \mathrm{H}_{70} \mathrm{O}_{22}$ (5.918)

$957.51003 \quad \mathrm{C}_{48} \mathrm{H}_{78} \mathrm{O}_{19}(3.735)$

$895.43802 \quad \mathrm{C}_{45} \mathrm{H}_{68} \mathrm{O}_{18}(5.283)$ $851.44613^{b} \quad \mathrm{C}_{44} \mathrm{H}_{68} \mathrm{O}_{16}$ (3.136)

$955.49392 \quad \mathrm{C}_{48} \mathrm{H}_{76} \mathrm{O}_{19}(3.261)$
$\mathrm{MS}^{2}$ [971.49]: 809 (100)[M-Hex-H] $]^{-}, 791$ (1) $\left[\mathrm{M}-\mathrm{Hex}-\mathrm{H}_{2} \mathrm{O}-\mathrm{H}\right]^{-}, 765$ (1)[M-Hex- $\mathrm{CO}_{2^{-}}$

$\mathrm{H}]^{-}, 629$ (2)[M-Hex-Hex- $\left.\mathrm{H}_{2} \mathrm{O}-\mathrm{H}\right]^{-}, 603$ (1)[M-Hex-Hex-CO $\left.{ }_{2}-\mathrm{H}\right]^{-}, 585$ (1)[M-Hex-Hex$\left.\mathrm{CO}_{2}-\mathrm{H}_{2} \mathrm{O}-\mathrm{H}\right]^{-}, 539(1), 471(1)[\mathrm{Agly}-\mathrm{H}]^{-}$

$\mathrm{MS}^{3}[971.49 \rightarrow 809]: 765(6)\left[\mathrm{M}-\mathrm{Hex}-\mathrm{CO}_{2}-\mathrm{H}\right]^{-}, 747(6)\left[\mathrm{M}-\mathrm{Hex}-\mathrm{CO}_{2}-\mathrm{H}_{2} \mathrm{O}-\mathrm{H}\right]^{-}, 647$ (1)[MHex-Hex-H] $]^{-}, 629$ (100)[M-Hex-Hex- $\left.\mathrm{H}_{2} \mathrm{O}-\mathrm{H}\right]^{-}, 611$ (2)[M-Hex-Hex- $\left.\mathrm{H}_{2} \mathrm{O}-\mathrm{H}_{2} \mathrm{O}-\mathrm{H}\right]^{-}, 603$ (27) $\left[\mathrm{M}-\mathrm{Hex}-\mathrm{Hex}-\mathrm{CO}_{2}-\mathrm{H}\right]^{-}, 585$ (24)[M-Hex-Hex-CO $\left.\mathrm{CO}_{2}-\mathrm{H}_{2} \mathrm{O}-\mathrm{H}\right]^{-}, 567$ (5)[M-Hex-Hex- $\mathrm{CO}_{2}$ $\left.\mathrm{H}_{2} \mathrm{O}-\mathrm{H}_{2} \mathrm{O}-\mathrm{H}\right]^{-}, 553$ (17), 539 (9) [M-Hex-Hex-108-H] $]^{-}, 471$ (100)[Agly-H $]^{-}, 453$ (17)

$\mathrm{MS}^{2}$ [1103.57]: $1085(100)\left[\mathrm{M}-\mathrm{H}_{2} \mathrm{O}-\mathrm{H}\right]^{-}, 1041(60)\left[\mathrm{M}-\mathrm{H}_{2} \mathrm{O}-\mathrm{CO}_{2}-\mathrm{H}\right]^{-}, 957$ (9)[M-dHex$\mathrm{H}]^{-}, 913$ (7) [M-dHex- $\left.\mathrm{CO}_{2}-\mathrm{H}\right]^{-}, 895$ (33) [M-dHex- $\left.\mathrm{H}_{2} \mathrm{O}-\mathrm{CO}_{2}-\mathrm{H}\right]^{-}, 795$ (2) [M-dHex-Hex$\mathrm{H}^{-}, 777$ (31)[M-dHex-Hex- $\left.\mathrm{H}_{2} \mathrm{O}-\mathrm{H}\right]^{-}, 759$ (16)[M-dHex-Hex- $\left.\mathrm{H}_{2} \mathrm{O}-\mathrm{H}_{2} \mathrm{O}-\mathrm{H}\right]^{-}, 620$ (2), 571 (1), 457 (1) [Agly-H $]^{-}$

$\mathrm{MS}^{3}[1103.57 \rightarrow 1085]: 1067(18)\left[\mathrm{M}-\mathrm{H}_{2} \mathrm{O}-\mathrm{H}_{2} \mathrm{O}-\mathrm{H}\right]^{-}, 1041(100)\left[\mathrm{M}-\mathrm{H}_{2} \mathrm{O}-\mathrm{CO}_{2}-\mathrm{H}\right]^{-}, 895$ (19) $\left[\mathrm{M}-\mathrm{dHex}-\mathrm{H}_{2} \mathrm{O}-\mathrm{CO}_{2}-\mathrm{H}\right]^{-}, 759$ (54) $\left[\mathrm{M}-\mathrm{dHex}-\mathrm{Hex}-\mathrm{H}_{2} \mathrm{O}-\mathrm{H}_{2} \mathrm{O}-\mathrm{H}\right]^{-}, 619$ (11)

(MS): $983(21)[\mathrm{M}-\mathrm{H}]^{-}, 895(21)\left[\mathrm{M}-\mathrm{CO}_{2}-\mathrm{CO}_{2}-\mathrm{H}\right]^{-}, 734(11)\left[\mathrm{M}^{-} \mathrm{CO}_{2}-\mathrm{CO}_{2}-\mathrm{Hex}-\mathrm{H}\right]^{-}, 691$ (100)[M-malonyl-Hex- $\left.\mathrm{CO}_{2}-\mathrm{H}\right]^{-}, 649$ (18)[M-malonyl-Hex-malonyl-H] $]^{-}, 631$ (62)[Mmalonyl-Hex-malonyl- $\left.\mathrm{H}_{2} \mathrm{O}-\mathrm{H}\right]^{-}, 487$ (39) $[\text { Agly-H }]^{-}$

$\mathrm{MS}^{2}\left[\right.$ 997.43]: $953(51)\left[\mathrm{M}-\mathrm{CO}_{2}-\mathrm{H}\right]^{-}, 909(100)\left[\mathrm{M}^{-} \mathrm{CO}_{2}-\mathrm{CO}_{2}-\mathrm{H}\right]^{-}, 733(6), 705(24)[\mathrm{M}-$ malonyl-Hex- $\left.\mathrm{CO}_{2}-\mathrm{H}\right]^{-}, 691(26)$

$\mathrm{MS}^{3}[997.43 \rightarrow 909]: 891(4)\left[\mathrm{M}-\mathrm{CO}_{2}-\mathrm{CO}_{2}-\mathrm{H}_{2} \mathrm{O}-\mathrm{H}\right]^{-}, 865(6)\left[\mathrm{M}-\mathrm{CO}_{2}-\mathrm{CO}_{2}-\mathrm{CO}_{2}-\mathrm{H}\right]^{-}, 849$

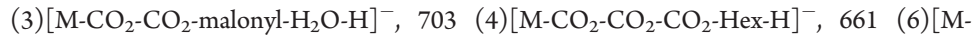

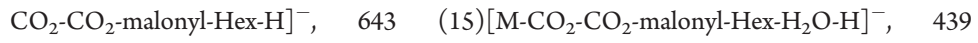
(100) $\left[\text { Agly- } \mathrm{CO}_{2}-\mathrm{H}_{2} \mathrm{O}-\mathrm{H}\right]^{-}$

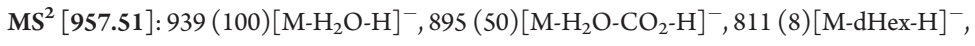
767 (3) [M-dHex- $\left.\mathrm{CO}_{2}-\mathrm{H}\right]^{-}, 749$ (29) [M-dHex- $\left.\mathrm{H}_{2} \mathrm{O}-\mathrm{CO}_{2}-\mathrm{H}\right]^{-}, 649$ (1) [M-dHex-Hex-H] $]^{-}$, 631 (29) [M-dHex-Hex- $\left.\mathrm{H}_{2} \mathrm{O}-\mathrm{H}\right]^{-}, 613$ (15) $\left[\mathrm{M}-\mathrm{dHex}-\mathrm{Hex}-\mathrm{H}_{2} \mathrm{O}-\mathrm{H}_{2} \mathrm{O}-\mathrm{H}\right]^{-}, 541$ (23)[MdHex-Hex-108-H $]^{-}, 473(8)[\text { Agly-H }]^{-}$

$\mathbf{M S}^{3}\left[\mathbf{9 5 7 . 5 1 \rightarrow 9 3 9 ] : ~} 895(100)\left[\mathrm{M}-\mathrm{H}_{2} \mathrm{O}-\mathrm{CO}_{2}-\mathrm{H}\right]^{-}, 749(14)\left[\mathrm{M}-\mathrm{dHex}-\mathrm{H}_{2} \mathrm{O}-\mathrm{CO}_{2}-\mathrm{H}\right]^{-}, 613\right.$ (61) $\left[\mathrm{M}-\mathrm{dHex}-\mathrm{Hex}-\mathrm{H}_{2} \mathrm{O}-\mathrm{H}_{2} \mathrm{O}-\mathrm{H}\right]^{-}, 541$ (62) $[\mathrm{M}-\mathrm{dHex}-\mathrm{Hex}-108-\mathrm{H}]^{-}$

MS $^{3}[957.51 \rightarrow 895]: 749(100)\left[\mathrm{M}-\mathrm{dHex}-\mathrm{H}_{2} \mathrm{O}-\mathrm{CO}_{2}-\mathrm{H}\right]^{-}, 587$ (5) $\left[\mathrm{M}-\mathrm{dHex}-\mathrm{Hex}-\mathrm{H}_{2} \mathrm{O}-\mathrm{CO}_{2}-\right.$ $\mathrm{H}]^{-}, 473(13)[\text { Agly-H }]^{-}$

$\mathrm{MS}^{2}$ [851.45]: 809 (29)[M-malonyl-H] $]^{-}, 791$ (27)[M-malonyl- $\left.\mathrm{H}_{2} \mathrm{O}-\mathrm{H}\right]^{-}, 689$ (82)[M-Hex$\left.\mathrm{CO}_{2}-\mathrm{H}\right]^{-}, 629$ (84)[M-malonyl-Hex- $\left.\mathrm{H}_{2} \mathrm{O}-\mathrm{H}\right]^{-}, 585$ (100)[M-malonyl-Hex- $\left.\mathrm{CO}_{2}-\mathrm{H}_{2} \mathrm{O}-\mathrm{H}\right]^{-}$, 471 (4) $[\text { Agly-H }]^{-}$

MS $^{3}[851.45 \rightarrow 585]: 513(100), 471(13)[\text { Agly-H }]^{-}$

MS $^{3}$ [851.45 $\left.\rightarrow 629\right]: 629(100)\left[\mathrm{M}-\text { malonyl- } \mathrm{H}_{2} \mathrm{O}-\mathrm{H}\right]^{-}, 513(54), 471(78)[\text { Agly-H }]^{-}, 453$ (45), 405 (64), 393 (74), 387 (22)

$\mathrm{MS}^{2}$ [955.49]: $937(100)\left[\mathrm{M}-\mathrm{H}_{2} \mathrm{O}-\mathrm{H}\right]^{-}, 911(34)\left[\mathrm{M}-\mathrm{CO}_{2}-\mathrm{H}\right]^{-}, 893(68)\left[\mathrm{M}-\mathrm{H}_{2} \mathrm{O}-\mathrm{CO}_{2}{ }^{-}\right.$ $\mathrm{H}^{-}, \quad 809(30)[\mathrm{M}-\mathrm{dHex}-\mathrm{H}]^{-}, 747$ (20)[M-dHex- $\left.\mathrm{H}_{2} \mathrm{O}-\mathrm{CO}_{2}-\mathrm{H}\right]^{-}, 689$ (8), 629 (48)[MdHex-Hex- $\left.\mathrm{H}_{2} \mathrm{O}-\mathrm{H}\right]^{-}, 611$ (8)[M-dHex-Hex- $\left.\mathrm{H}_{2} \mathrm{O}-\mathrm{H}_{2} \mathrm{O}-\mathrm{H}\right]^{-}, 539$ (14)[M-dHex-Hex-108$\mathrm{H}]^{-}, 471(17)[\text { Agly-H }]^{-}$

$\mathrm{MS}^{2}$ [865.43]: 823 (3)[M-malonyl- $]^{-}, 821(4)\left[\mathrm{M}-\mathrm{CO}_{2}-\mathrm{CO}_{2}-\mathrm{H}\right]^{-}, 805$ (28)[M-malonyl$\left.\mathrm{H}_{2} \mathrm{O}-\mathrm{H}\right]^{-}, 761$ (4) $\left[\mathrm{M} \text {-malonyl- } \mathrm{CO}_{2}-\mathrm{H}_{2} \mathrm{O}-\mathrm{H}\right]^{-}, 703(8)\left[\mathrm{M}-\mathrm{CO}_{2}-\mathrm{Hex}-\mathrm{H}\right]^{-}, 643(100)[\mathrm{M}-$ malonyl-Hex- $\left.\mathrm{H}_{2} \mathrm{O}-\mathrm{H}\right]^{-}, 599$ (35) [M-malonyl-Hex- $\left.\mathrm{CO}_{2}-\mathrm{H}_{2} \mathrm{O}-\mathrm{H}\right]^{-}, 485$ (1) $[\text { Agly-H }]^{-}$ MS $^{3}[865.43 \rightarrow 643]: 509(100), 485(64)[\text { Agly-H }]^{-}, 467$ (41), 421 (41) 
Table 1. Continued

\begin{tabular}{|c|c|c|c|}
\hline $\begin{array}{l}\text { Peak numbers and tentative } \\
\text { identification of the observed saponin }\end{array}$ & $\mathrm{s}[\mathrm{M}-\mathrm{H}]^{-}$ & $\begin{array}{l}\text { Formula } \\
(\delta \mathrm{ppm})\end{array}$ & FT-ICR MS ${ }^{n}: m / z$ (\% base peak) \\
\hline 41 Hex-Hederagenin & 633.40242 & $\mathrm{C}_{36} \mathrm{H}_{58} \mathrm{O}_{9}(2.547)$ & $\begin{array}{l}\mathrm{MS}^{2}[633.40]: 615(33)\left[\mathrm{M}-\mathrm{H}_{2} \mathrm{O}-\mathrm{H}\right]^{-}, 513(15), 471(100)[\text { Agly-H}]^{-}, 453 \text { (24), } 405 \text { (24), } \\
393 \text { (39) }\end{array}$ \\
\hline $\begin{array}{l}42 \text { Hex-Hex-HexA- } \\
\text { Soyasapogenol E }\end{array}$ & 955.49420 & $\mathrm{C}_{48} \mathrm{H}_{76} \mathrm{O}_{19}(3.554)$ & 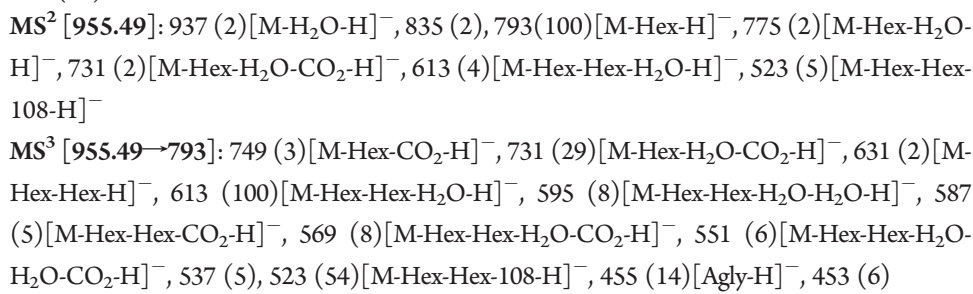 \\
\hline $\begin{array}{l}43 \text { Malonyl-Hex-Malonyl-Hex- } \\
\text { Hederagenin }\end{array}$ & $\begin{array}{l}967.45877 \\
879.47798^{b}\end{array}$ & $\mathrm{C}_{48} \mathrm{H}_{72} \mathrm{O}_{20}(4.498)$ & 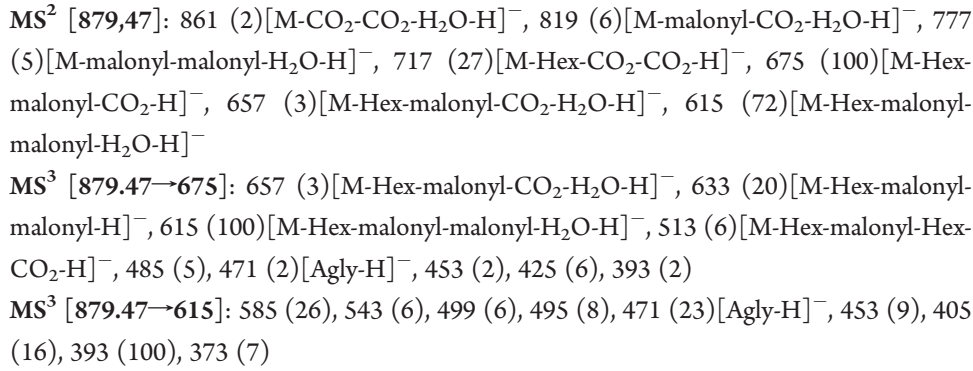 \\
\hline 44 Hex-Pen-Hederagenin & 765.44543 & $\mathrm{C}_{71} \mathrm{H}_{66} \mathrm{O}_{13}(3.088)$ & $\begin{array}{l}\text { MS }^{2}[765.45]: 603(100)[\mathrm{M}-\mathrm{Hex}-\mathrm{H}]^{-}, 585(7)\left[\mathrm{M}-\mathrm{Hex}-\mathrm{H}_{2} \mathrm{O}-\mathrm{H}\right]^{-}, 471(6)[\text { Agly-H}]^{-} \\
\mathbf{M S}^{3}[765.45 \rightarrow 603]: 471(100)[\text { Agly-H}]^{-}, 423(14)\end{array}$ \\
\hline $\begin{array}{l}45 \text { dHex-Hex-Hex- } \\
\text { Soyasapogenol E }\end{array}$ & 925.51905 & $\mathrm{C}_{48} \mathrm{H}_{78} \mathrm{O}_{17}(2.620)$ & $\begin{array}{l}\text { MS }^{2}\left[\text { 925.52]: } 779 \text { (100) }[\mathrm{M}-\mathrm{dHex}-\mathrm{H}]^{-}, 761(13)\left[\mathrm{M}-\mathrm{dHex}-\mathrm{H}_{2} \mathrm{O}-\mathrm{H}\right]^{-}, 617(7)[\mathrm{M}-\mathrm{dHex}-\right. \\
\mathrm{Hex}-\mathrm{H}]^{-}, 599(1)\left[\mathrm{M}-\mathrm{dHex}-\mathrm{Hex}-\mathrm{H}_{2} \mathrm{O}-\mathrm{H}\right]^{-}, 455(1)[\text { Agly-H}]^{-} \\
\text {MS }^{3}[\mathbf{9 2 5 . 5 2} \rightarrow 779]: 617(100)[\mathrm{M}-\mathrm{dHex}-\mathrm{Hex}-\mathrm{H}]^{-}, 599(4)\left[\mathrm{M}-\mathrm{dHex}-\mathrm{Hex}-\mathrm{H}_{2} \mathrm{O}-\mathrm{H}\right]^{-}, 455 \\
\text { (9) }[\text { Agly-H}]^{-}\end{array}$ \\
\hline 46 dHex-Hex-HexA-Hederagenin & 955.49417 & $\mathrm{C}_{48} \mathrm{H}_{76} \mathrm{O}_{19}(3.523)$ & 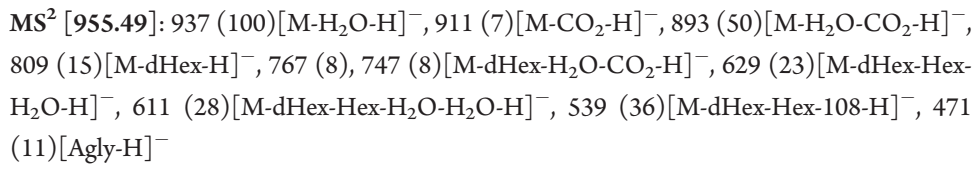 \\
\hline $\begin{array}{l}47 \text { Malonyl-Hex-Hex-HexA- } \\
\text { Soyasapogenol E }\end{array}$ & $\begin{array}{l}1041.49582 \\
997.50288^{b}\end{array}$ & $\mathrm{C}_{51} \mathrm{H}_{78} \mathrm{O}_{22}(4.438)$ & 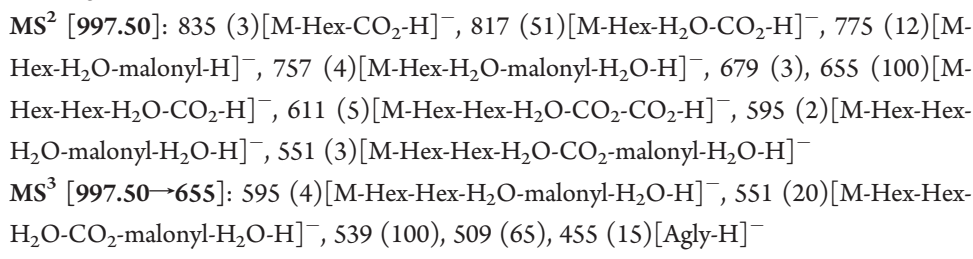 \\
\hline 48 dHex-Hex-Hex-Aglycone A & 955.49454 & $\mathrm{C}_{48} \mathrm{H}_{76} \mathrm{O}_{19}(3.910)$ & 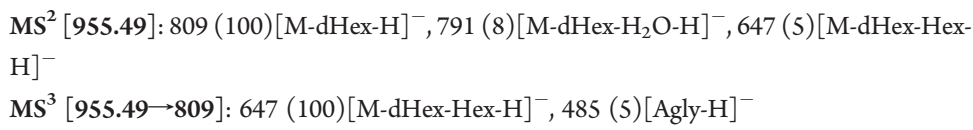 \\
\hline 49 dHex-Hex-HexA-Bayogenin & 971.48812 & $\mathrm{C}_{48} \mathrm{H}_{76} \mathrm{O}_{20}(2.473)$ & 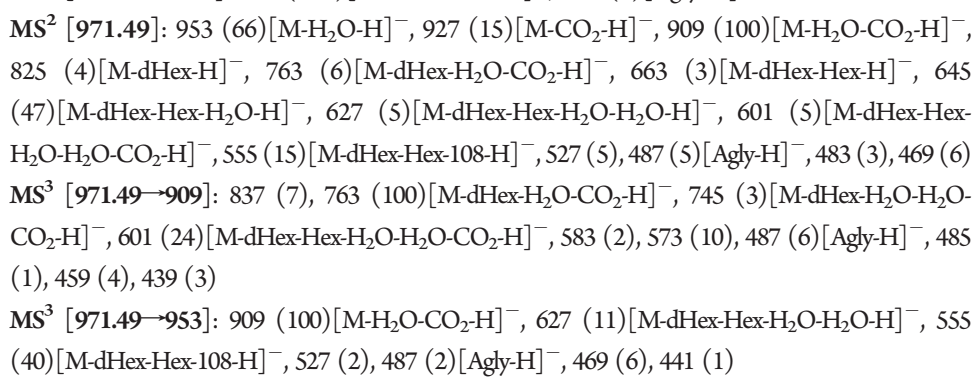 \\
\hline $50 \mathrm{dHex}-H e x-H e x A-H e d e r a g e n i n$ & 955.49245 & $\mathrm{C}_{48} \mathrm{H}_{76} \mathrm{O}_{19}(1.723)$ & $\begin{array}{l}\mathrm{MS}^{2}[955.49]: 937(100)\left[\mathrm{M}-\mathrm{H}_{2} \mathrm{O}-\mathrm{H}\right]^{-}, 893(35)\left[\mathrm{M}-\mathrm{H}_{2} \mathrm{O}-\mathrm{CO}_{2}-\mathrm{H}\right]^{-}, 747(30)[\mathrm{M}-\mathrm{dHex}- \\
\left.\mathrm{H}_{2} \mathrm{O}-\mathrm{CO}_{2}-\mathrm{H}\right]^{-}, 629(41)\left[\mathrm{M}-\mathrm{dHex}-\mathrm{Hex}-\mathrm{H}_{2} \mathrm{O}-\mathrm{H}\right]^{-}, 611(22)\left[\mathrm{M}-\mathrm{dHex}-\mathrm{Hex}-\mathrm{H}_{2} \mathrm{O}-\mathrm{H}_{2} \mathrm{O}-\mathrm{H}\right]^{-}, \\
539(21)[\mathrm{M}-\mathrm{dHex}-\mathrm{Hex}-108-\mathrm{H}]^{-}, 471(18)[\text { Agly-H}]^{-}, 451(4), 307(4)\end{array}$ \\
\hline 51 dHex-Hex-Hex-Bayogenin & 957.50866 & $\mathrm{C}_{48} \mathrm{H}_{78} \mathrm{O}_{19}(2.304)$ & $\begin{array}{l}\text { MS }^{2}[\mathbf{9 5 7 . 5 0}]: 939(2)\left[\mathrm{M}-\mathrm{H}_{2} \mathrm{O}-\mathrm{H}\right]^{-}, 811(100)[\mathrm{M}-\mathrm{dHex}-\mathrm{H}]^{-}, 793(6)\left[\mathrm{M}-\mathrm{dHex}-\mathrm{H}_{2} \mathrm{O}-\mathrm{H}\right]^{-}, \\
649(12)[\mathrm{M}-\mathrm{dHex}-\mathrm{Hex}-\mathrm{H}]^{-}, 631(2)\left[\mathrm{M}-\mathrm{dHex}-\mathrm{Hex}-\mathrm{H}_{2} \mathrm{O}-\mathrm{H}\right]^{-}, 487(2)[\mathrm{Agly}-\mathrm{H}]^{-} \\
\mathbf{M S}^{3}[\mathbf{9 5 7 . 5 0} \rightarrow \mathbf{8 1 1}]: 649(100)[\mathrm{M}-\mathrm{dHex}-\mathrm{Hex}-\mathrm{H}]^{-}, 631(12)\left[\mathrm{M}-\mathrm{dHex}-\mathrm{Hex}-\mathrm{H}_{2} \mathrm{O}-\mathrm{H}\right]^{-}, 487 \\
(56)[\text { Agly-H}]^{-}\end{array}$ \\
\hline
\end{tabular}


Table 1. Continued

\begin{tabular}{|c|c|c|c|}
\hline $\begin{array}{l}\text { Peak numbers and tentative } \\
\text { identification of the observed saponin }\end{array}$ & as $[\mathrm{M}-\mathrm{H}]^{-}$ & $\begin{array}{l}\text { Formula } \\
(\delta \mathrm{ppm})\end{array}$ & FT-ICR MS ${ }^{n}: m / z$ (\% base peak) \\
\hline 52 Hex-HexA-Bayogenin & 825.43024 & $\mathrm{C}_{42} \mathrm{H}_{66} \mathrm{O}_{16}(3.162)$ & $\begin{array}{l}\mathbf{M S}^{2}[825.43]: 645(100)\left[\mathrm{M}-\mathrm{Hex}-\mathrm{H}_{2} \mathrm{O}-\mathrm{H}\right]^{-}, 619(28)\left[\mathrm{M}-\mathrm{Hex}-\mathrm{CO}_{2}-\mathrm{H}\right]^{-}, 601(20)[\mathrm{M}-\mathrm{Hex}- \\
\left.\mathrm{H}_{2} \mathrm{O}-\mathrm{CO}_{2}-\mathrm{H}\right]^{-}, 569(11), 487(78)[\text { Agly-H}]^{-}, 455(8) \\
\mathbf{M S}^{3}[825.43 \rightarrow 645]: 557(11), 487(11)[\text { Agly-H}]^{-}, 469(47), 439(100) \\
\mathbf{M S}^{3}[825.43 \rightarrow 487]: 467(67), 455(72), 437(15), 409(100), 393(10), 391(15)\end{array}$ \\
\hline $\begin{array}{l}53 \text { Malonyl-dHex-Hex-HexA- } \\
\text { Soyasapogenol B } \\
\text { (Pisumsaponin I) }\end{array}$ & 1027.51636 & $\mathrm{C}_{51} \mathrm{H}_{80} \mathrm{O}_{21}$ (4.308) & 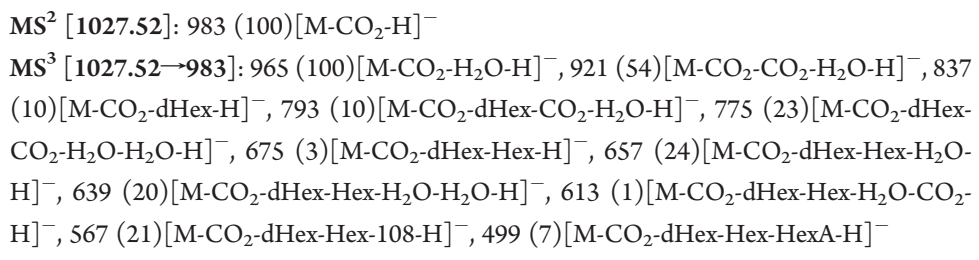 \\
\hline 54 Hex-HexA-Soyasapogenol E & 793.43923 & $\mathrm{C}_{42} \mathrm{H}_{66} \mathrm{O}_{14}(1.575)$ & $\begin{array}{l}\text { MS }^{2}[793.44]: 673(8), 631(100)[\mathrm{M}-\mathrm{Hex}-\mathrm{H}]^{-}, 613(13)\left[\mathrm{M}-\mathrm{Hex}-\mathrm{H}_{2} \mathrm{O}-\mathrm{H}\right]^{-}, 569(8)[\mathrm{M}- \\
\left.\mathrm{Hex}-\mathrm{H}_{2} \mathrm{O}-\mathrm{CO}_{2}-\mathrm{H}\right]^{-} \\
\mathbf{M S}^{3}[793.44 \rightarrow 631]: 613(4)\left[\mathrm{M}-\mathrm{Hex}-\mathrm{H}_{2} \mathrm{O}-\mathrm{H}\right]^{-}, 555(3), 497(2), 455(100)[\text { Agly-H}]^{-}\end{array}$ \\
\hline $\begin{array}{l}55 \text { Malonyl-dHex-Hex- } \\
\text { Medicagenic acid }\end{array}$ & $\begin{array}{l}895.43821 \\
851.44654^{b}\end{array}$ & $\begin{array}{l}\mathrm{C}_{45} \mathrm{H}_{68} \mathrm{O}_{18}(5.496) \\
\mathrm{C}_{44} \mathrm{H}_{68} \mathrm{O}_{16}(3.617)\end{array}$ & $\begin{array}{l}\mathrm{MS}^{2}[851.45]: 809(49)[\mathrm{M}-\text { malonyl-H}]^{-}, 791(8)\left[\mathrm{M}-\text { malonyl- } \mathrm{H}_{2} \mathrm{O}-\mathrm{H}\right]^{-}, 689(37)[\mathrm{M}-\mathrm{Hex}- \\
\left.\mathrm{CO}_{2}-\mathrm{H}\right]^{-}, 629(48)\left[\mathrm{M}-\text { malonyl-Hex- } \mathrm{H}_{2} \mathrm{O}-\mathrm{H}\right]^{-}, 585(100)\left[\mathrm{M}-\text { malonyl-Hex }-\mathrm{H}_{2} \mathrm{O}-\mathrm{CO}_{2}-\mathrm{H}\right]^{-}, \\
439(9)\left[\text { Agly- } \mathrm{CO}_{2}-\mathrm{H}_{2} \mathrm{O}-\mathrm{H}\right]^{-}\end{array}$ \\
\hline 56 Hex-Hex-Bayogenin & 811.45028 & $\mathrm{C}_{42} \mathrm{H}_{68} \mathrm{O}_{15}(2.138)$ & $\begin{array}{l}\mathbf{M S}^{2}[811.45]: 649(100)[\mathrm{M}-\mathrm{Hex}-\mathrm{H}]^{-}, 631(8)\left[\mathrm{M}-\mathrm{Hex}-\mathrm{H}_{2} \mathrm{O}-\mathrm{H}\right]^{-}, 487(58)[\text { Agly-H }]^{-} \\
\mathbf{M S}^{3}[811.45 \rightarrow 649]: 487(100)[\text { Agly-H}]^{-}, 439(1)\end{array}$ \\
\hline 57 dHex-Hex-Hex-Aglycone A & 955.49230 & $\mathrm{C}_{48} \mathrm{H}_{76} \mathrm{O}_{19}(1.566)$ & 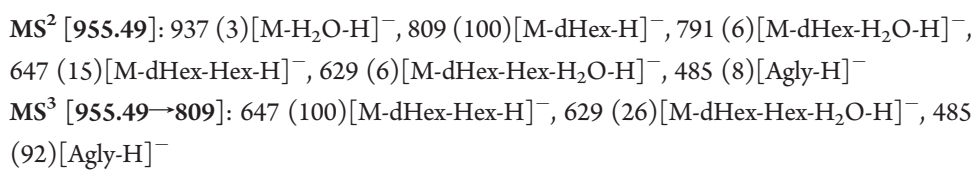 \\
\hline 58 Hex-Hex-Bayogenin & 811.45207 & $\mathrm{C}_{42} \mathrm{H}_{68} \mathrm{O}_{15}$ & $\begin{array}{l}\mathbf{M S}^{2}[811.45]: 649(100)[\mathrm{M}-\mathrm{Hex}-\mathrm{H}]^{-}, 631(31)\left[\mathrm{M}-\mathrm{Hex}-\mathrm{H}_{2} \mathrm{O}-\mathrm{H}\right]^{-}, 487(98)[\text { Agly-H}]^{-} \\
\mathbf{M S}^{3}[811.45 \rightarrow 649]: 487(100)[\text { Agly-H}]^{-}, 439(1)\end{array}$ \\
\hline 59 Hex-HexA-Bayogenin & 825.43143 & $\mathrm{C}_{42} \mathrm{H}_{66} \mathrm{O}_{16}(4.386)$ & $\begin{array}{l}\mathbf{M S}^{2}[825.43]: 645(100)\left[\mathrm{M}-\mathrm{Hex}-\mathrm{H}_{2} \mathrm{O}-\mathrm{H}\right]^{-}, 601 \quad(17)\left[\mathrm{M}-\mathrm{Hex}-\mathrm{H}_{2} \mathrm{O}-\mathrm{CO}_{2}-\mathrm{H}\right]^{-}, 487 \\
(6)[\text { Agly-H}]^{-}, 439(1) \\
\mathbf{M S}^{3}[825.43 \rightarrow 645]: 487(100)[\text { Agly-H}]^{-}, 467(2), 455(5), 439(9) \\
\mathbf{M S}^{3}[\mathbf{8 2 5 . 4 3 \rightarrow 6 0 1}]: 529(100), 487(8)[\text { Agly-H}]^{-}\end{array}$ \\
\hline 60 3-Glc-Glc-Medicagenic acid & 825.43148 & $\mathrm{C}_{42} \mathrm{H}_{66} \mathrm{O}_{16}(4.446)$ & $\begin{array}{l}\mathrm{MS}^{2}[825.43]: 663(1)[\mathrm{M}-\mathrm{Glc}-\mathrm{H}]^{-}, 601(1)\left[\mathrm{M}-\mathrm{Glc}_{-} \mathrm{H}_{2} \mathrm{O}-\mathrm{CO}_{2}-\mathrm{H}\right]^{-}, 439(100)\left[\mathrm{Agly}^{-} \mathrm{CO}_{2^{-}}\right. \\
\left.\mathrm{H}_{2} \mathrm{O}-\mathrm{H}\right]^{-} \\
\mathbf{M S}^{3}[825.43 \rightarrow 439]: 393(61), 391(100), 375(25)\end{array}$ \\
\hline 61 dHex-Hex-Hex-Hederagenin & 941.51621 & $\mathrm{C}_{48} \mathrm{H}_{78} \mathrm{O}_{18}(4.961)$ & $\begin{array}{l}\text { MS }^{2}[\mathbf{9 4 1 . 5 1}]: 795(100)[\text { M-dHex-H }]^{-}, 777(10)\left[\text { M-dHex- }{ }_{2} \mathrm{O}-\mathrm{H}\right]^{-}, 633(13)[\text { M-dHex- } \\
\left.\mathrm{Hex}^{-}\right]^{-}, 615(2)\left[\mathrm{M}-\mathrm{dHex}-\mathrm{Hex}-\mathrm{H}_{2} \mathrm{O}-\mathrm{H}\right]^{-}, 471(1)[\text { Agly-H}]^{-} \\
\text {MS }^{3}[\mathbf{9 4 1 . 5 1} \rightarrow \mathbf{7 9 5}]: 633(100)[\mathrm{M}-\mathrm{dHex}-\mathrm{Hex}-\mathrm{H}]^{-}, 615(5)\left[\mathrm{M}-\mathrm{dHex}-\mathrm{Hex}-\mathrm{H}_{2} \mathrm{O}-\mathrm{H}\right]^{-}, 471 \\
(24)[\text { Agly-H}]^{-} \\
\text {MS }^{3}\left[\mathbf{9 4 1 . 5 1 \rightarrow 6 3 3 ] : ~} 471(100)[\text { Agly-H}]^{-}\right.\end{array}$ \\
\hline 62 Malonyl-Hex-Hex-B & $\begin{array}{l}897.45213 \\
853.46093^{b}\end{array}$ & $\begin{array}{l}\mathrm{C}_{45} \mathrm{H}_{70} \mathrm{O}_{18}(3.556) \\
\mathrm{C}_{44} \mathrm{H}_{70} \mathrm{O}_{16}(2.132)\end{array}$ & 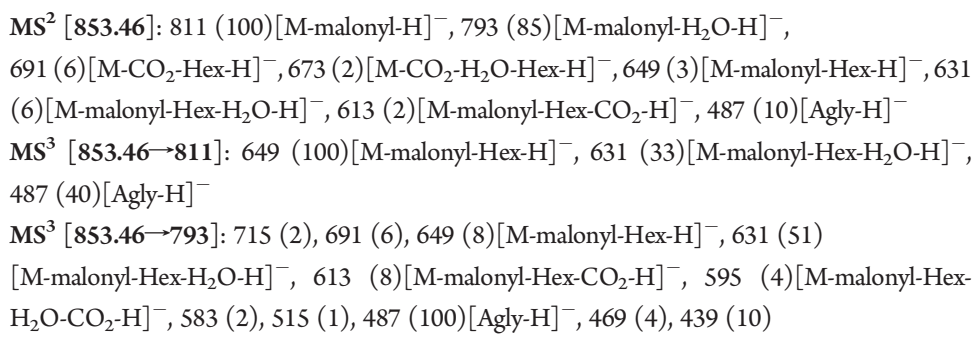 \\
\hline 63 Hex-HexA-Hederagenin & 809.43693 & $\mathrm{C}_{42} \mathrm{H}_{66} \mathrm{O}_{15}(4.985)$ & $\begin{array}{l}\mathrm{MS}^{2}[809.44]: 747(5)\left[\mathrm{M}-\mathrm{H}_{2} \mathrm{O}-\mathrm{CO}_{2}-\mathrm{H}\right]^{-}, 647(2)[\mathrm{M}-\mathrm{Hex}-\mathrm{H}]^{-}, 629(100)\left[\mathrm{M}-\mathrm{Hex}-\mathrm{H}_{2} \mathrm{O}-\right. \\
\mathrm{H}]^{-}, 603(11)\left[\mathrm{M}-\mathrm{Hex}-\mathrm{CO}_{2}-\mathrm{H}\right]^{-}, 585(14)\left[\mathrm{M}-\mathrm{Hex}-\mathrm{H}_{2} \mathrm{O}-\mathrm{CO}_{2}-\mathrm{H}\right]^{-}, 567(2)\left[\mathrm{M}-\mathrm{Hex}-\mathrm{H}_{2} \mathrm{O}-\right. \\
\left.\mathrm{H}_{2} \mathrm{O}-\mathrm{CO}_{2}-\mathrm{H}\right]^{-}, 553(5), 539(2)[\mathrm{M}-\mathrm{Hex}-108-\mathrm{H}]^{-}, 471(15)[\text { Agly-H}]^{-}, 469(6), 453(1), 451 \\
(1), 439(8), 423(2)\end{array}$ \\
\hline 64 Hex-HexA-Aglycone A & 823.41390 & $\mathrm{C}_{42} \mathrm{H}_{66} \mathrm{O}_{16}(2.113)$ & 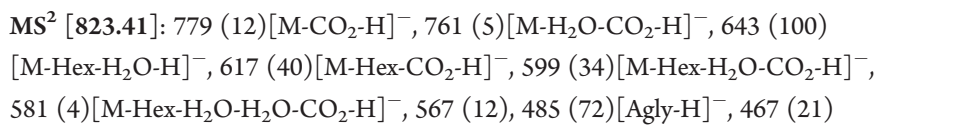 \\
\hline
\end{tabular}


Table 1. Continued

\begin{tabular}{|c|c|c|c|}
\hline $\begin{array}{l}\text { Peak numbers and tentative } \\
\text { identification of the observed saponins }\end{array}$ & s $[\mathrm{M}-\mathrm{H}]^{-}$ & $\begin{array}{l}\text { Formula } \\
(\delta \mathrm{ppm})\end{array}$ & FT-ICR MS ${ }^{n}: m / z$ (\% base peak) \\
\hline 65 Pen-Hex-Hex-Aglycone B & 925.48149 & $\mathrm{C}_{47} \mathrm{H}_{74} \mathrm{O}_{18}(1.352)$ & 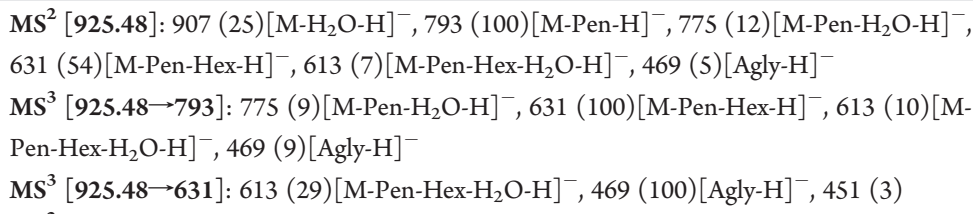 \\
\hline 66 Hex-Hex-Aglycone A & 809.43647 & $\mathrm{C}_{42} \mathrm{H}_{66} \mathrm{O}_{15}(4.417)$ & $\begin{array}{l}\mathbf{M S}^{2}[809.44]: 647(99)[\mathrm{M}-\mathrm{Hex}-\mathrm{H}]^{-}, 629(27)\left[\mathrm{M}-\mathrm{Hex}-\mathrm{H}_{2} \mathrm{O}-\mathrm{H}\right]^{-}, 485(100)[\text { Agly-H}]^{-} \\
\mathbf{M S}^{3}[809.44 \rightarrow 485]: 467(100), 455(21), 439(11), 423(40), 421(61), 403(48), 391(11), \\
363(10), 347(18) \\
\mathbf{M S}^{3}[809.44 \rightarrow 647]: 485(100)[\text { Agly-H }]^{-}\end{array}$ \\
\hline 67 Hex-Medicagenic acid & 663.37661 & $\mathrm{C}_{36} \mathrm{H}_{56} \mathrm{O}_{11}(2.448)$ & $\begin{array}{l}\mathbf{M S}^{2}[663.38]: 487(6)\left[\text { Agly- } \mathrm{H}_{2} \mathrm{O}-\mathrm{H}\right]^{-}, 439(100)\left[\text { Agly }^{-} \mathrm{CO}_{2}-\mathrm{H}_{2} \mathrm{O}-\mathrm{H}\right]^{-} \\
\mathbf{M S}^{3}[663.38 \rightarrow 439]: 393(61), 391(100), 375(11)\end{array}$ \\
\hline $\begin{array}{l}68 \text { dHex-Hex-HexA- } \\
\text { Soyasapogenol E }\end{array}$ & 939.49979 & $\mathrm{C}_{48} \mathrm{H}_{76} \mathrm{O}_{18}(4.152)$ & $\begin{array}{l}\mathrm{MS}^{2}[939.50]: 921(100)\left[\mathrm{M}-\mathrm{H}_{2} \mathrm{O}-\mathrm{H}\right]^{-}, 877(24)\left[\mathrm{M}-\mathrm{H}_{2} \mathrm{O}-\mathrm{CO}_{2}-\mathrm{H}\right]^{-}, 793(11)[\mathrm{M}-\mathrm{dHex}- \\
\mathrm{H}^{-}, 749(5)\left[\mathrm{M}-\mathrm{dHex}-\mathrm{CO}_{2}-\mathrm{H}\right]^{-}, 731(18)\left[\mathrm{M}-\mathrm{dHex}-\mathrm{H}_{2} \mathrm{O}-\mathrm{CO}_{2}-\mathrm{H}\right]^{-}, 613(14)[\mathrm{M}-\mathrm{dHex}- \\
\left.\mathrm{Hex}-\mathrm{H}_{2} \mathrm{O}-\mathrm{H}\right]^{-}, 595(17)\left[\mathrm{M}-\mathrm{dHex}-\mathrm{Hex}-2 \mathrm{H}_{2} \mathrm{O}-\mathrm{H}\right]^{-}, 523(11)[\mathrm{M}-\mathrm{dHex}-\mathrm{Hex}-108-\mathrm{H}]^{-}, 465 \\
(24), 455(8)[\text { Agly-H}]^{-} \\
\mathrm{MS}^{3}[939.50 \rightarrow 921]: 877(100)\left[\mathrm{M}-\mathrm{H}_{2} \mathrm{O}-\mathrm{CO}_{2}-\mathrm{H}\right]^{-}, 731(44)\left[\mathrm{M}-\mathrm{dHex}-\mathrm{H}_{2} \mathrm{O}-\mathrm{CO}_{2}-\mathrm{H}\right]^{-}, 595 \\
(35)\left[\mathrm{M}-\mathrm{dHex}-\mathrm{Hex}-2 \mathrm{H}_{2} \mathrm{O}-\mathrm{H}\right]^{-}, 523(14)[\mathrm{M}-\mathrm{dHex}-\mathrm{Hex}-108-\mathrm{H}]^{-}, 465(45)\end{array}$ \\
\hline 69 dHex-Hex-HexA-Hederagenin & 955.49569 & $\mathrm{C}_{48} \mathrm{H}_{76} \mathrm{O}_{19}(5.114)$ & $\begin{array}{l}\mathrm{MS}^{2}[955.49]: 937(100)\left[\mathrm{M}-\mathrm{H}_{2} \mathrm{O}-\mathrm{H}\right]^{-}, 893(63)\left[\mathrm{M}-\mathrm{H}_{2} \mathrm{O}-\mathrm{CO}_{2}-\mathrm{H}\right]^{-}, 809(14)[\mathrm{M}-\mathrm{dHex}- \\
\mathrm{H}]^{-}, 765(23)\left[\mathrm{M}-\mathrm{dHex}-\mathrm{CO}_{2}-\mathrm{H}\right]^{-}, 747(19)\left[\mathrm{M}-\mathrm{dHex}-\mathrm{H}_{2} \mathrm{O}-\mathrm{CO}_{2}-\mathrm{H}\right]^{-}, 629(18)[\mathrm{M}-\mathrm{dHex}- \\
\left.\mathrm{Hex}-\mathrm{H}_{2} \mathrm{O}-\mathrm{H}\right]^{-}, 611(19)\left[\mathrm{M}-\mathrm{dHex}-\mathrm{Hex}-2 \mathrm{H}_{2} \mathrm{O}-\mathrm{H}\right]^{-}, 539(21)[\mathrm{M}-\mathrm{dHex}-\mathrm{Hex}-108-\mathrm{H}]^{-}, 471 \\
(8)[\text { Agly-H}]^{-}, 469(3)\end{array}$ \\
\hline 70 Hex-Bayogenin & 649.39741 & $\mathrm{C}_{36} \mathrm{H}_{58} \mathrm{O}_{10}(2.599)$ & $\begin{array}{l}\operatorname{MS}^{2}[649.39]: 487(100)[\text { Agly-H }]^{-}, 409(3), 391(2) \\
\text { MS }^{3}[649.39 \rightarrow 487]: 467(29), 455(14), 421(35), 409 \text { (100), } 403 \text { (17), } 393 \text { (34), } 391 \text { (52), } \\
379(17)\end{array}$ \\
\hline 71 Hex-HexA-Hederagenin & 809.43534 & $\mathrm{C}_{42} \mathrm{H}_{66} \mathrm{O}_{15}(3.021)$ & $\begin{array}{l}\mathbf{M S}^{2}[\mathbf{8 0 9 . 4 4}]: 629(100)\left[\mathrm{M}-\mathrm{Hex}-\mathrm{H}_{2} \mathrm{O}-\mathrm{H}\right]^{-}, \quad 585 \quad(11)\left[\mathrm{M}-\mathrm{Hex}-\mathrm{H}_{2} \mathrm{O}-\mathrm{CO}_{2}-\mathrm{H}\right]^{-}, 471 \\
(2)[\text { Agly-H}]^{-} \\
\mathbf{M S}^{3}[\mathbf{8 0 9 . 4 4 \rightarrow 6 2 9}]: 499(12), 471(100)[\text { Agly-H}]^{-}, 469(11), 439(12) \\
\mathbf{M S}^{3}[\mathbf{8 0 9 . 4 4 \rightarrow 5 8 5}]: 513(100), 499(2), 471(5)[\text { Agly-H}]^{-}\end{array}$ \\
\hline 72 Malonyl-Hex-Medicagenic acid & $\begin{array}{l}749.37838 \\
705.38773^{b}\end{array}$ & $\begin{array}{l}\mathrm{C}_{39} \mathrm{H}_{58} \mathrm{O}_{14}(4.003) \\
\mathrm{C}_{38} \mathrm{H}_{58} \mathrm{O}_{12}(3.089)\end{array}$ & 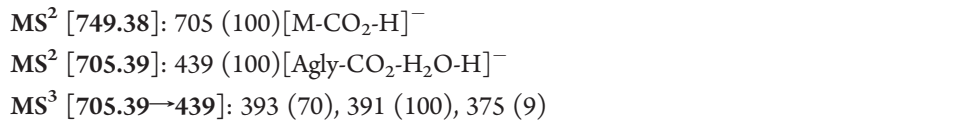 \\
\hline $\begin{array}{l}73 \text { Pen-Hex-HexA- } \\
\text { Soyasapogenol B }\end{array}$ & 927.49925 & $\mathrm{C}_{47} \mathrm{H}_{76} \mathrm{O}_{18}(3.624)$ & $\begin{array}{l}\mathrm{MS}^{2}[927.50]: 909(100)\left[\mathrm{M}-\mathrm{H}_{2} \mathrm{O}-\mathrm{H}\right]^{-}, 865(14)\left[\mathrm{M}-\mathrm{CO}_{2}-\mathrm{H}_{2} \mathrm{O}-\mathrm{H}\right]^{-}, 795(50)[\mathrm{M}-\mathrm{Pen}-\mathrm{H}]^{-}, \\
\left.733 \text { (34)[M-Pen- } \mathrm{CO}_{2}-\mathrm{H}_{2} \mathrm{O}-\mathrm{H}\right]^{-}, 633(3)[\mathrm{M}-\mathrm{Pen}-\mathrm{Hex}-\mathrm{H}]^{-}, 615(44)\left[\mathrm{M}-\mathrm{Pen}-\mathrm{Hex}-\mathrm{H}_{2} \mathrm{O}-\right. \\
\mathrm{H}]^{-}, \quad 597 \quad(65)\left[\mathrm{M}-\mathrm{Pen}-\mathrm{Hex}-\mathrm{H}_{2} \mathrm{O}-\mathrm{H}_{2} \mathrm{O}-\mathrm{H}\right]^{-}, \quad 571 \quad(4)\left[\mathrm{M}-\mathrm{Pen}-\mathrm{Hex}-\mathrm{CO}_{2}-\mathrm{H}_{2} \mathrm{O}-\mathrm{H}\right]^{-}, \quad 525 \\
(72)[\mathrm{M}-\mathrm{Pen}-\mathrm{Hex}-108-\mathrm{H}]^{-}, 457(15)[\text { Agly-H}]^{-}\end{array}$ \\
\hline 74 Hex-HexA-Aglycone A & 823.41450 & $\mathrm{C}_{42} \mathrm{H}_{66} \mathrm{O}_{16}(2.842)$ & 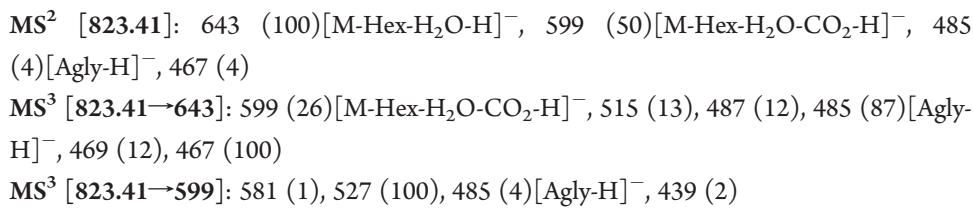 \\
\hline 75 Malonyl-Hex-Bayogenin & $\begin{array}{l}735.39924 \\
691.40869^{b}\end{array}$ & $\begin{array}{l}\mathrm{C}_{39} \mathrm{H}_{60} \mathrm{O}_{13}(4.003) \\
\mathrm{C}_{38} \mathrm{H}_{60} \mathrm{O}_{11}(3.477)\end{array}$ & $\begin{array}{l}\text { MS }^{2}[691.41]: 649(10)[\text { M-malonyl-H }]^{-}, 631(100)\left[\mathrm{M}-\text { malonyl- } \mathrm{H}_{2} \mathrm{O}-\mathrm{H}\right]^{-}, 487(2)[\text { Agly-H}]^{-} \\
\mathrm{MS}^{3}[691.41 \rightarrow 631]: 601(100), 487(10)[\text { Agly-H}]^{-}, 469(20), 421(6), 409(18), 393(10), 391 \\
(10) \\
\text { MS }^{3}[691.41 \rightarrow 649]: 487(100)[\text { Agly-H}]^{-}, 439(1)\end{array}$ \\
\hline $\begin{array}{l}76 \text { Malonyl-Hex-HexA- } \\
\text { Hederagenin }\end{array}$ & $\begin{array}{l}895.43874 \\
851.44633^{b}\end{array}$ & $\begin{array}{l}\mathrm{C}_{45} \mathrm{H}_{68} \mathrm{O}_{18}(6.088) \\
\mathrm{C}_{44} \mathrm{H}_{68} \mathrm{O}_{16}(3.371)\end{array}$ & $\begin{array}{l}\left.\mathrm{MS}^{2}[851.45]: 809 \text { (1)[M-malonyl-H }\right]^{-}, 791(2)\left[\mathrm{M}-\text { malonyl- } \mathrm{H}_{2} \mathrm{O}-\mathrm{H}\right]^{-}, 671 \text { (39) }[\mathrm{M}-\mathrm{Hex}- \\
\left.\left.\mathrm{C}_{2} \mathrm{H}_{2} \mathrm{O}-\mathrm{H}_{2} \mathrm{O}-\mathrm{H}\right]^{-}, 627 \text { (14)[M-Hex-malonyl- } \mathrm{H}_{2} \mathrm{O}-\mathrm{H}\right]^{-}, 611 \text { (11) }\left[\mathrm{M}-\mathrm{Hex}-\text { malonyl- } \mathrm{CO}_{2}-\right. \\
\mathrm{H}]^{-}, 585(9), 567(13), 499(100), 471(4)[\text { Agly-H}]^{-}\end{array}$ \\
\hline $\begin{array}{l}77 \text { 3-Rha-Gal-GlcA- } \\
\text { Soyasapogenol B }\end{array}$ & 941.51540 & $\mathrm{C}_{48} \mathrm{H}_{78} \mathrm{O}_{18}(4.101)$ & 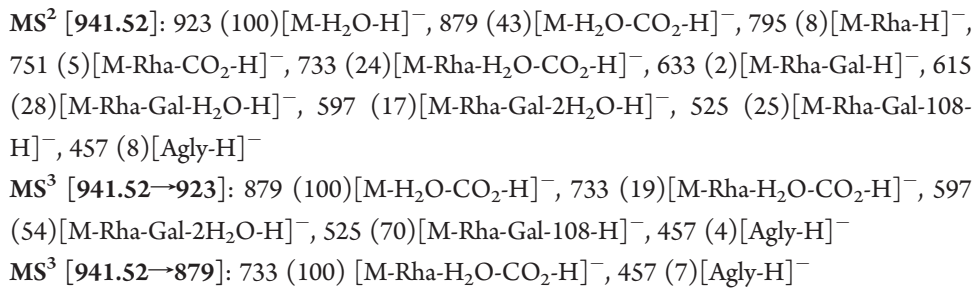 \\
\hline
\end{tabular}


Table 1. Continued

Peak numbers and tentative

Formula

identification of the observed saponins $[\mathrm{M}-\mathrm{H}]^{-} \quad(\delta \mathrm{ppm})$

FT-ICR $\mathrm{MS}^{n}: m / z$ (\% base peak)

78 Rha-Gal-GlcA-Soyasapogenol E $939.49939 \mathrm{C}_{48} \mathrm{H}_{76} \mathrm{O}_{18}(3.726) \quad \mathbf{M S}^{2}[\mathbf{9 3 9 . 5 0}]: 921(100)\left[\mathrm{M}-\mathrm{H}_{2} \mathrm{O}-\mathrm{H}\right]^{-}, 877(42)\left[\mathrm{M}-\mathrm{H}_{2} \mathrm{O}-\mathrm{CO}{ }^{-}-\mathrm{H}\right]^{-}, 793(8)[\mathrm{M}-\mathrm{Rha}-\mathrm{H}]^{-}$, 749 (1) $\left[\mathrm{M}-\mathrm{Rha}-\mathrm{CO}_{2}-\mathrm{H}\right]^{-}, 731$ (41) $\left[\mathrm{M}-\mathrm{Rha}-\mathrm{H}_{2} \mathrm{O}-\mathrm{CO}_{2}-\mathrm{H}\right]^{-}, 613$ (47)[M-Rha-Gal- $\mathrm{H}_{2} \mathrm{O}-$ $\mathrm{H}]^{-}, 595$ (29) $\left[\mathrm{M}-\mathrm{Rh} \text { a-Gal-2 } \mathrm{H}_{2} \mathrm{O}-\mathrm{H}\right]^{-}, 523$ (64)[M-Rha-Gal-108-H $]^{-}, 455(5)[\text { Agly-H }]^{-}$ $\mathbf{M S}^{3}[939.50 \rightarrow 921]: 877(78)\left[\mathrm{M}-\mathrm{H}_{2} \mathrm{O}-\mathrm{CO}_{2}-\mathrm{H}\right]^{-}, 731(26)\left[\mathrm{M}-\mathrm{Rha}-\mathrm{H}_{2} \mathrm{O}-\mathrm{CO}_{2}-\mathrm{H}\right]^{-}, 595$ (36) [M-Rha-Gal-2 $\left.\mathrm{H}_{2} \mathrm{O}-\mathrm{H}\right]^{-}, 523(100)[\mathrm{M}-\mathrm{Rha}-\mathrm{Gal}-108-\mathrm{H}]^{-}, 455(6)[$ Agly-H] MS $^{3}[\mathbf{9 3 9 . 5 0} \rightarrow 523]: 455(91)[\text { Agly-H] }]^{-}, 453(100), 439(13)$

79 HexA-Hederagenin $\quad 647.38199 \quad \mathrm{C}_{36} \mathrm{H}_{56} \mathrm{O}_{10}(3.726) \quad \mathbf{M S}^{2}[647.38]: 629(5), 587(7), 571(18), 527(4), 499(3), 471(100)[\text { Agly-H] }]^{-}, 439(7)$ MS $^{3}[647.38 \rightarrow 471]: 439(18), 405(43), 393(100)$

${ }^{a}$ HexA, uronic acid, such as glucuronic acid or galacturonic acid; Hex, hexose, such as glucose or galactose; dHex, 6-deoxyhexose, such as rhamnose or furanose; Pen, pentose, such as arabinose or xylose; GlcA, glucuronic acid; Glc, glucose; Gal, galactose; Rha, rhamnose. ${ }^{b}$ Ion resulting from in-source fragmentation.

interchanged with dependent IT-MS ${ }^{n}$ scan events, existing of an $\mathrm{MS}^{2}$ and two $\mathrm{MS}^{3}$ scans. In the $\mathrm{MS}^{2}$ scan, the most abundant ion in the previous full MS scan was fragmented. The two most abundant daughter ions in every $\mathrm{MS}^{2}$ scan event were subjected to a dependent $\mathrm{MS}^{3}$ scan event. In addition, in-source fragmentation data were included in the identification process.

Identification of Saponins. Saponins are amphipathic glycosides consisting of a lipophilic aglycone or sapogenin covalently linked to one (monodesmosidic) or more (di- or tridesmosidic) sugar chains via an ether or ester glycosidic bond. The sugar residues are mainly hexoses (e.g., glucose and galactose), 6-deoxyhexoses (e.g., rhamnose and furanose), pentoses (e.g., arabinose and xylose), and uronic acids (e.g., glucuronic acid and galacturonic acid). As reported before, upon collision-induced dissociation (CID) under negative ESI-MS, saponins undergo glycosidic cleavages, retaining the charge at the reducing terminus (i.e., the fragment that contains the aglycone). ${ }^{21,22}$ The detected (grand)daughter ions in the $\mathrm{MS}^{n}$ spectra provide structural information about the sugar residues and aglycone of the fragmented saponin.

Figure 1 illustrates the identification of a hitherto unknown saponin. As indicated in Figure 1A, the unknown saponin showed an $[\mathrm{M}-\mathrm{H}]^{-}$anion at $\mathrm{m} / z$ 945.50965. Because saponins consist of the elements $\mathrm{C}, \mathrm{H}$, and $\mathrm{O}$, three different molecular formulas are possible within an error range of 5 ppm: $\mathrm{C}_{65} \mathrm{H}_{69} \mathrm{O}_{6}(\delta \mathrm{ppm}=$ $-0.331), \mathrm{C}_{40} \mathrm{H}_{81} \mathrm{O}_{24}(\delta \mathrm{ppm}=-2.830)$, and $\mathrm{C}_{47} \mathrm{H}_{77} \mathrm{O}_{19}$ $(\delta \mathrm{ppm}=3.380)$. To determine the correct molecular formula, the abundance of the isotopes was considered. In nature, stable elemental isotopes occur in a stable ratio. For instance, ${ }^{13} \mathrm{C}$ occurs at approximately $1.11 \%$ of the most frequent carbon isotope, ${ }^{12} \mathrm{C}$. Similarly, ${ }^{2} \mathrm{H}$ and ${ }^{17} \mathrm{O}$ occur at 0.015 and $0.038 \%$ of ${ }^{1} \mathrm{H}$ and ${ }^{16} \mathrm{O}$, respectively. Hence, the observed monoisotopic quasi-molecular ions $\left(\mathrm{M}_{0}\right)$ are always accompanied by additional isotope ions $(M+1, M+2, \ldots)$ (Figure $1 B)$, of which the relative abundance depends on the elemental composition of the compound. The predicted relative abundance of the $M+1$ ions of the three possible molecular formulas was 73.41, 46.53, and $54.05 \%$, respectively. For the unknown saponin, the observed relative abundance of the $M+1$ ion was $53.63 \%$ (Figure 1B), indicating that the molecular formula corresponding to the $[\mathrm{M}-$ $\mathrm{H}]^{-}$anion is $\mathrm{C}_{47} \mathrm{H}_{77} \mathrm{O}_{19}(\delta \mathrm{ppm}=3.380)$. The sugar residues and the aglycone could be identified from the $\mathrm{MS}^{n}$ spectra. For this saponin, $\mathrm{MS}^{2}$ fragmentation led to the generation of five daughter ions, resulting from the loss of water $(-18 \mathrm{Da})$ and/or one or more hexose $(-162 \mathrm{Da})$ and/or pentose $(-132 \mathrm{Da})$ residues. The smallest daughter ion, at $m / z 489$, represents the aglycone ion, $[\text { Agly }-\mathrm{H}]^{-}$, resulting from the loss of a pentose and two hexose moieties (Figure 1C). Based on this rationale, tens of other saponins present in the extract of $M$. truncatula hairy roots could tentatively be identified. All identified saponins and their $\mathrm{MS}^{n}$ data are listed in Table 1.

Cross-Ring Cleavage of Uronic Acid Residues. Saponins are reported to undergo only glycosidic cleavages upon CID under negative ESI-MS. ${ }^{21}$ However, in this study, we observed a recurrent fragmentation pattern for saponins that contain a uronic acid residue, indicating a cross-ring cleavage of the uronic acid residue upon CID, as illustrated with the $\mathrm{MS}^{2}$ spectrum observed for 3-Rha-Gal-GlcA-soyasapogenol B (Figure 2A,B). The anions with the highest relative intensity, observed at $\mathrm{m} / z$ 923 and 879 , correlated to the neutral losses of $18\left(\mathrm{H}_{2} \mathrm{O}\right)$ and 62 $\mathrm{Da}\left(\mathrm{H}_{2} \mathrm{O}+\mathrm{CO}_{2}\right)$, respectively. A similar pattern was observed after the loss of the rhamnose residue $(\mathrm{m} / z$ 795), and the ions observed at $\mathrm{m} / z 751$ and 733 correlated to an additional loss of $44\left(\mathrm{CO}_{2}\right)$ and $62 \mathrm{Da}\left(\mathrm{H}_{2} \mathrm{O}+\mathrm{CO}_{2}\right)$, respectively. Furthermore, three more characteristic peaks were observed at $m / z 615,597$, and 525, corresponding to a loss of the galactose residue, and an additional loss of $18\left(\mathrm{H}_{2} \mathrm{O}\right), 36\left(\mathrm{H}_{2} \mathrm{O}+\mathrm{H}_{2} \mathrm{O}\right)$, and $108 \mathrm{Da}$. The latter loss can only be explained by a cross-ring cleavage of the uronic acid residue. Cross-ring cleavages of glycosides are chargeremote fragmentations, i.e., fragmentations that are not initiated by the charge position. Because of the low internal energy of negative ions, cross-ring cleavages of glycosides do not readily occur. $^{21}$ An exception are those that are linked via an ester bond as in, for example, feruloylated oligosaccharides ${ }^{23,24}$ or sinapoyl glucose. ${ }^{25}$ Sinapoyl glucose serves as a UV protectant in Arabidopsis, and upon CID in the negative ionization mode, losses of 60,90 , and $120 \mathrm{Da}$ are observed, ${ }^{25}$ which correspond to a ${ }^{0,4} \mathrm{X}$, ${ }^{0,3} \mathrm{X}$, and ${ }^{0,2} \mathrm{X}$ cross-ring cleavage, respectively (see legend of Figure 2 for explanation on nomenclature). However, in this study, the cross-ring cleavage of the uronic acid residue should be charge-driven, because the carboxylic group of the uronic acid bears the negative charge. The loss of $108 \mathrm{Da}$ might be due to the loss of water followed by an additional loss of a $\mathrm{C}_{3} \mathrm{H}_{6} \mathrm{O}_{3}$ fragment of $90 \mathrm{Da}$ or by the loss of a $\mathrm{C}_{3} \mathrm{H}_{8} \mathrm{O}_{4}$ fragment involving ${ }^{1,4} \mathrm{X}$ cross-ring cleavage of the uronic acid residue (Figure $2 \mathrm{C}$ ). This typical fragmentation pattern was observed in 20 compounds and aided in the tentative identification of hitherto unknown saponins. By means of this methodology, a total of 79 saponins present in the $M$. truncatula hairy root extracts were identified (Table 1). However, $\mathrm{MS}^{n}$ does not provide enough information for absolute structural characterization of the metabolites; hence, the identifications remain tentative. 
A ctr1b RT: 33.66 AV: 1 NL: $1.06 E 5$

T: ITMS - c ESI d Full ms2941.52@cid35.00 [245.00-955.00]

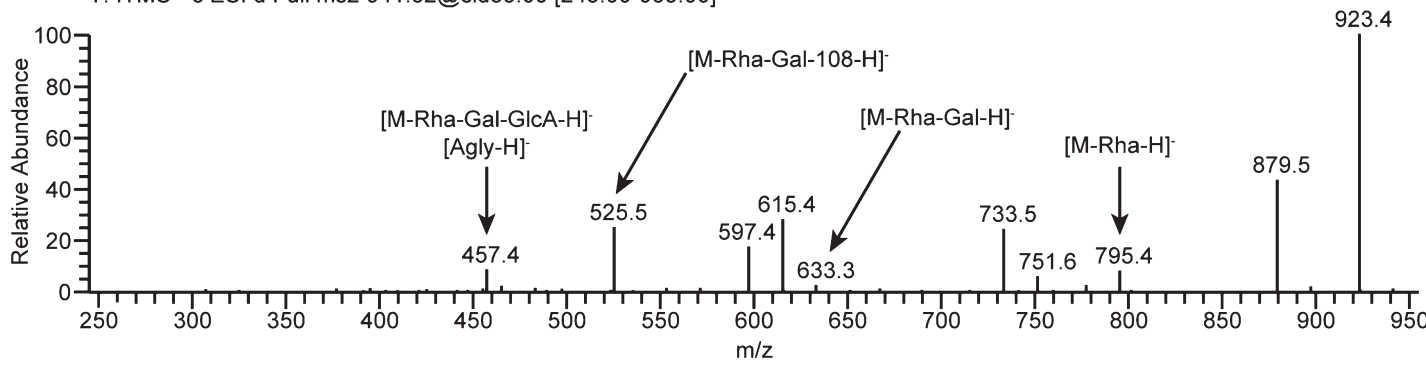

B

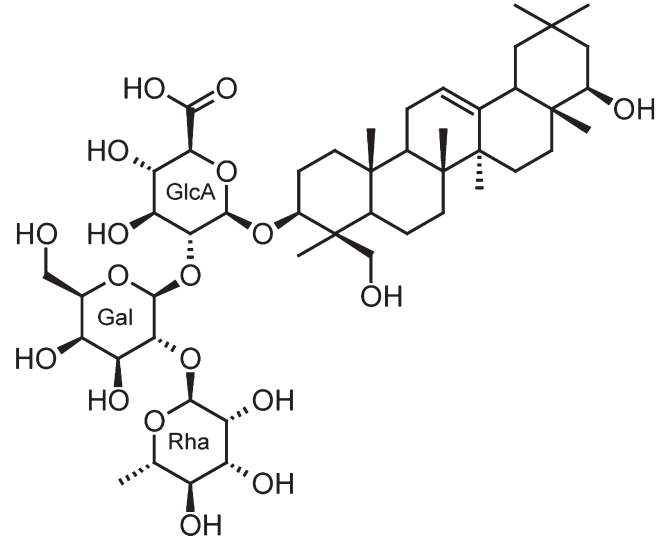

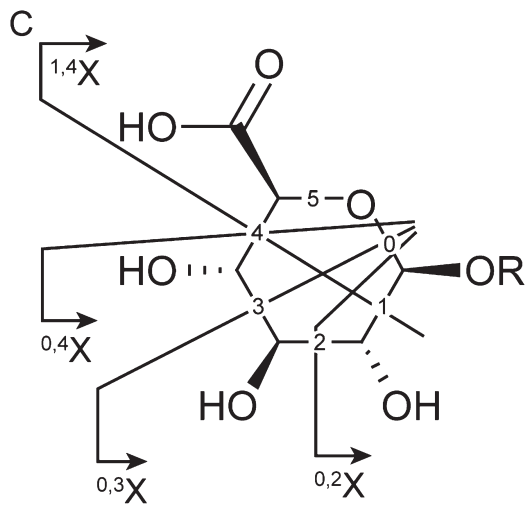

Figure 2. Cross-ring cleavage of uronic acid residues. (A) MS ${ }^{2}$ fragmentation of 3-Rha-Gal-GlcA-soyasapogenol B. (B) Structure of 3-Rha-Gal-GlcAsoyasapogenol B. (C) Nomenclature of the cross-ring fragmentation of glucuronic acid.

A ctr1b RT: 33.63 AV: 1 NL: $3.53 E 7$

T: FTMS - p ESI Full ms [120.00-1400.00]

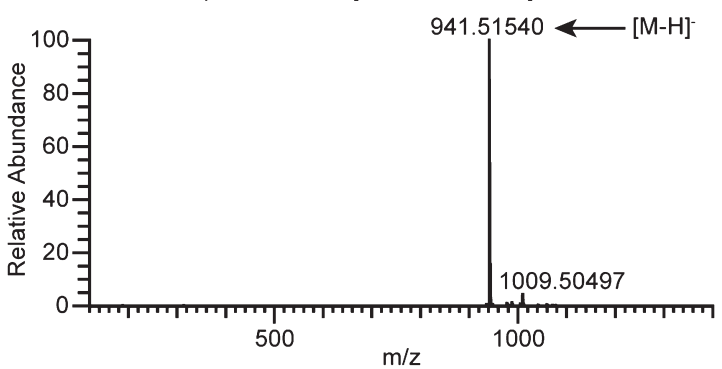

C ctr1b RT: 25.65 AV: 1 NL: 1.46E4

T: ITMS - c ESI d Full ms2 825.43@cid35.00 [215.00-840.00]

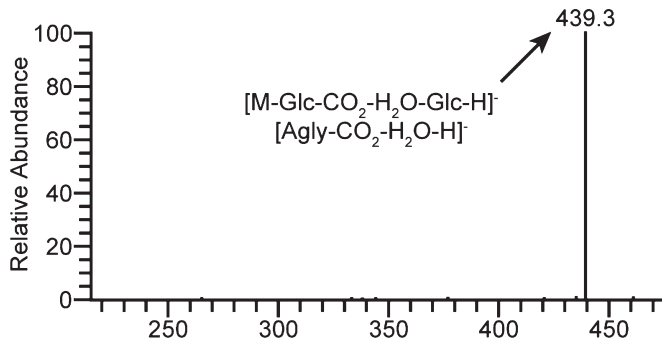

B ctr1b RT: 25.80 AV: 1 NL: 7.48E6

T: FTMS - p ESI Full ms [120.00-1400.00]

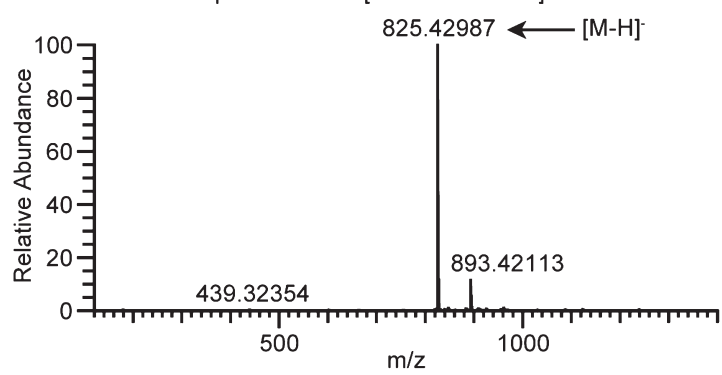

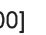

$\left.\left[\mathrm{M}-\mathrm{GlC}-\mathrm{CO}_{2}-\mathrm{H}_{2} \mathrm{O}-\mathrm{H}\right]\right]\left[\mathrm{M}-\mathrm{GIC}-\mathrm{CO}_{2}-\mathrm{H}\right]$

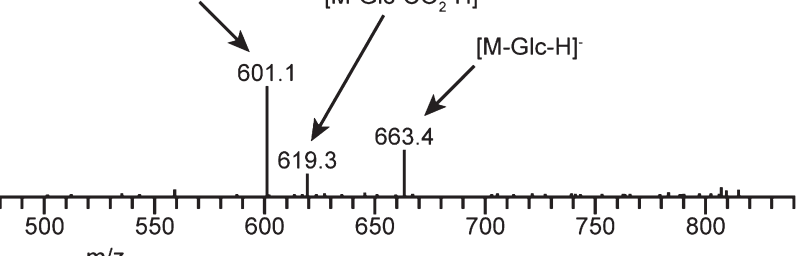

Figure 3. LC ESI FT-ICR MS chromatograms. (A) MS scan of peak at $t_{\mathrm{R}} 33.63 \mathrm{~min}$ (3-Rha-Gal-GlcA-soyasapogenol B). (B) MS scan of peak at $t_{\mathrm{R}} 25.80 \mathrm{~min}$ (3-Glc-28-Glc-medicagenic acid). (C) $\mathrm{MS}^{2}$ fragmentation of 3-Glc-28-Glc-medicagenic acid.

Most Abundant Compounds in M. truncatula Hairy Roots. In the metabolite extracts of $M$. truncatula hairy roots, the major peak, at a retention time $\left(t_{\mathrm{R}}\right)$ of $33.63 \mathrm{~min}$, yielded an $[\mathrm{M}-\mathrm{H}]^{-}$ anion at $m / z 941.51540\left(\mathrm{C}_{48} \mathrm{H}_{78} \mathrm{O}_{18}, \delta \mathrm{ppm}=4.101\right)$
(Figure 3A). In M. truncatula, two saponins have been described with the same molecular formula, Rha-Hex-Hex-hederagenin and soyasaponin I (3-Rha-Gal-GlcA-soyasapogenol B). ${ }^{7}$ The $\mathrm{MS}^{2}$ spectrum (Figure $2 \mathrm{~A}$ and Table 1 ) corresponded to 
<smiles>CC1(C)CCC2(C(=O)O)CCC3C(=CCC4C3CCC3C4CCC4C(C)(C)C(O)C(O)CC34C)CC12</smiles><smiles>CC1(C)CCC2(C(=O)O)CCC3C(=CCC4C3CCC3C4CCC4C(C)(C)C(O)CCC34C)C2C1</smiles><smiles>CC(C)CC1CCC2(C(=O)O)CCC(C)(C)CC2C2=CCC3C(CCC4C(C)(CO)C(O)C(O)CC34C)C21</smiles>

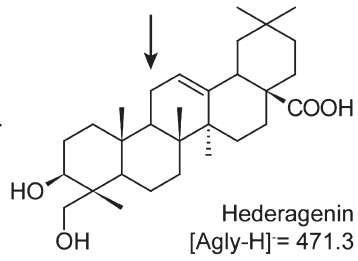

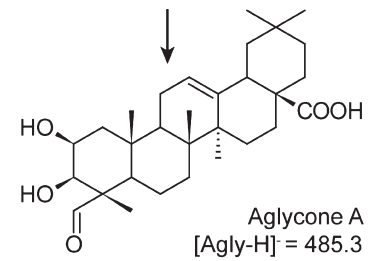

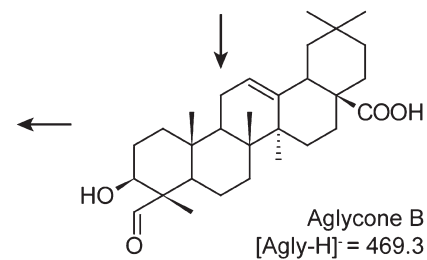<smiles>CCC12CC(O)C(O)C(C)(C(=O)O)C1CCC1C2CC=C2C3CC(C)(C)CCC3(C(=O)O)C(O)CC21C</smiles><smiles>CC1(C)CC(O)C2(C)CCC3C(=CCC4C5CCC6C(C)(C)C(O)CCC6(C)C5CCC34C)C2C1</smiles>

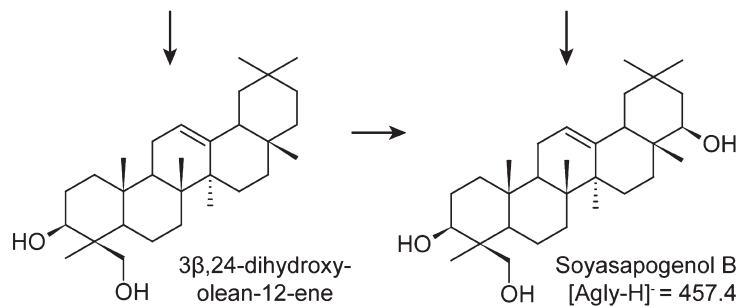

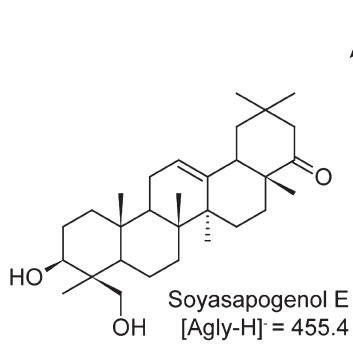

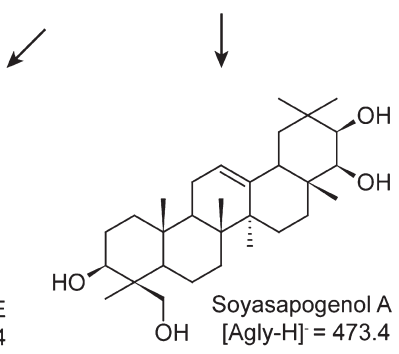

Figure 4. Theoretical biosynthetic pathway of sapogenins in $M$. truncatula and $\mathrm{m} / \mathrm{z}$ values of the observed aglycones in the hairy roots extract.

previously observed MS/MS data of soyasaponin $\mathrm{I},{ }^{26}$ allowing us to conclude that soyasaponin I is the most abundant saponin in the $M$. truncatula hairy roots extract. This is consistent with the previous observation that the major saponins in the roots of $M$. truncatula are soyasapogenol conjugates. ${ }^{7}$ The second most abundant compound, eluting at $25.80 \mathrm{~min}$, yielded an $[\mathrm{M}-$ $\mathrm{H}]^{-}$anion at $m / z 825.42987\left(\mathrm{C}_{42} \mathrm{H}_{66} \mathrm{O}_{16}, \delta \mathrm{ppm}=2.494\right)$ (Figure 3B). In the $\mathrm{MS}^{2}$ spectrum (Figure 3C), the base peak occurs at $m / z 439$, a fragment ion resulting from the decarboxylation and dehydration of the medicagenic acid aglycone ion. ${ }^{2}$ Additional anions were observed at $m / z 663,619$, and 601, correlating to the sequential loss of a hexose (162 Da), a $\mathrm{CO}_{2}$ (44 Da), and $\mathrm{H}_{2} \mathrm{O}(18 \mathrm{Da})$ molecule from the parent ion. This fragmentation pattern corresponded to the previously reported MS data of 3-Glc-28-Glc-medicagenic acid and 3-Glc-Glc-medicagenic acid. ${ }^{2}$ To annotate the detected compound, we scanned for other peaks containing an anion with an $\mathrm{m} / z$ value between 825.40 and 825.45 . Four additional isomers were detected, but only one $\left(t_{\mathrm{R}} 32.15 \mathrm{~min}\right)$ yielded a fragment ion at $\mathrm{m} / z 439$ associated with medicagenic acid. As 3-Glc-28-Glc-medicagenic acid has been reported to have a lower retention time than 3-GlcGlc-medicagenic acid, ${ }^{2}$ the peak at $t_{\mathrm{R}} 25.80 \mathrm{~min}$ has been annotated as 3-Glc-28-Glc-medicagenic acid, and the peak at $t_{\mathrm{R}}$ $32.15 \mathrm{~min}$ as 3-Glc-Glc-medicagenic acid.

Absence of Zanhic Acid Glycosides. A remarkable observation was the absence of zanhic acid glycosides in the list of identified saponins. In previous studies, zanhic acid was shown to be one of the major aglycone moieties in aerial parts of $M$. truncatula, ${ }^{8,9}$ and it was also reported to be present in other $M$. truncatula plant tissues, including roots. ${ }^{7}$ In a more recent study, however, zanhic acid could not be detected in M. truncatula roots. ${ }^{27}$ Similarly, no zanhic acid-containing compounds were detected in the hairy roots. Hairy root cultures are known to accumulate secondary metabolites that are normally produced in "natural" roots of differentiated plants. ${ }^{13,14,28}$ Hence, the absence of zanhic acid residues in hairy roots may indicate that this compound is produced only in the aerial parts of the plant. Consequently, the occurrence of the enzyme responsible for the $16 \alpha$-hydroxylation of the saponin backbone (Figure 4) might be restricted to the aerial parts of the plant. This finding might further support a tissue-specific role for the saponins in planta. Indeed, the saponin mixture from the aerial parts of the plant has a protective effect against herbivores, whereas that from the roots rather shows allelopathic and antimicrobial activities. ${ }^{29}$ Thus, the in vivo role of the zanhic acid glycosides might be related to antinutritional effects in the aerial parts of the plant. In addition to zanhic acid glycosides, aerial parts are particularly rich in medicagenic acid glycosides. ${ }^{7-9}$ Notably, none of the major medicagenic acid glycosides occurring in the aerial parts of the plant were found in the hairy roots, further underscoring the unique saponin composition of roots and aerial parts. In root extracts of greenhouse-grown $M$. truncatula plants, the presence of 31 saponins, mainly soyasapogenol conjugates, was shown. ${ }^{7}$ Here, we could confirm the presence of 18 of these 31 previously described saponins. For some of the remaining saponins of this list, we could detect the corresponding nominal masses, but the observed accurate masses and fragmentation data did not unambiguously support the proposed structures. Hence, we did not include them in Table 1 . However, in addition to the 18 previously described saponins, 61 other saponins were 
Table 2. Observed Aglycones in M. truncatula

\begin{tabular}{|c|c|c|}
\hline$m / z$ [observed ion] & Molecular formula & Tentative identification \\
\hline $455[\text { Agly-H }]^{-}$ & $\mathrm{C}_{30} \mathrm{H}_{48} \mathrm{O}_{3}$ & Soyasapogenol E \\
\hline $457[\text { Agly-H }]^{-}$ & $\mathrm{C}_{30} \mathrm{H}_{50} \mathrm{O}_{3}$ & Soyasapogenol B \\
\hline $469[\text { Agly-H }]^{-}$ & $\mathrm{C}_{30} \mathrm{H}_{46} \mathrm{O}_{4}$ & Aglycone B ( $3 \beta$-hydroxy-23-oxo-olean-12-en-28-oic acid) \\
\hline $471[\text { Agly-H }]^{-}$ & $\mathrm{C}_{30} \mathrm{H}_{48} \mathrm{O}_{4}$ & Hederagenin \\
\hline $473[\text { Agly-H }]^{-}$ & $\mathrm{C}_{30} \mathrm{H}_{50} \mathrm{O}_{4}$ & Soyasapogenol A \\
\hline $485[\text { Agly-H }]^{-}$ & $\mathrm{C}_{30} \mathrm{H}_{46} \mathrm{O}_{5}$ & Aglycone $\mathrm{A}$ ( $2 \beta, 3 \beta$-dihydroxy-23-oxo-olean-12-en-28-oic acid) \\
\hline $487[\text { Agly-H }]^{-}$ & $\mathrm{C}_{30} \mathrm{H}_{48} \mathrm{O}_{5}$ & Bayogenin \\
\hline $489[\text { Agly-H }]^{-}$ & $\mathrm{C}_{30} \mathrm{H}_{50} \mathrm{O}_{5}$ & Aglycone D \\
\hline $437\left[\text { Agly- } \mathrm{CO}_{2}-\mathrm{H}_{2} \mathrm{O}-\mathrm{H}\right]^{-}$ & $\mathrm{C}_{30} \mathrm{H}_{44} \mathrm{O}_{6}$ & Aglycone C \\
\hline $439\left[\text { Agly- } \mathrm{CO}_{2}-\mathrm{H}_{2} \mathrm{O}-\mathrm{H}\right]^{-}$ & $\mathrm{C}_{30} \mathrm{H}_{46} \mathrm{O}_{6}$ & Medicagenic acid \\
\hline
\end{tabular}

encountered in this study, all of which had not been detected before in M. truncatula. This result underscores the power of the applied FT-ICR MS platform in metabolome analysis.

Ten Different Aglycones. One prerequisite we applied to include the detected compounds in the list of tentatively identified saponins was the occurrence of an aglycone ion in the $\mathrm{MS}^{n}$ fragmentation data. As such, we could show that the 79 identified saponins present in the extract of $M$. truncatula hairy roots were glycosides of 10 different aglycones. The structures of the aglycones are given in Figure 4, and the observed aglycone ions, their predicted molecular formulas, and tentative identifications are listed in Table 2. For five of the aglycones, i.e., hederagenin, bayogenin, medicagenic acid, and soyasapogenols B and E (Figure 4), saponins containing the aglycone have been described before. ${ }^{2,7-9}$ A sixth aglycone yielded an $[\mathrm{Agly}-\mathrm{H}]^{-}$ion at $m / z 473$ (Figure 5A). Although this aglycone was only detected as a (grand)daughter ion with nominal $\mathrm{m} / z$ values, its molecular formula could be predicted by the accurate prediction of the molecular formula of the parent ion and the observed loss of sugar residues. With this method, the molecular formula was predicted to be $\mathrm{C}_{30} \mathrm{H}_{50} \mathrm{O}_{4}$, which corresponds to the molecular formula of soyasapogenol A. No saponins of $M$. truncatula that contain soyasapogenol A as the aglycone had been described, but the compound had been shown to occur in $M$. truncatula as a sapogenin. ${ }^{27}$ Hence, the two saponins containing this aglycone were tentatively identified as soyasapogenol A glycosides.

Furthermore, four other, unknown, aglycones were observed. Aglycone A was shown to occur in 10 of the tentatively identified saponins. It had an observed [Agly $-\mathrm{H}]^{-}$ion at $m / z 485$ (Figure 5C), and its molecular formula was calculated to be $\mathrm{C}_{30} \mathrm{H}_{46} \mathrm{O}_{5}$. An aglycone with this mass had been previously observed in $M$. truncatula, but its structure was not elucidated. ${ }^{2,7,30}$ Only one sapogenin with this molecular formula is known to occur in the genus Medicago, namely, $2 \beta, 3 \beta$-dihydroxy-23-oxoolean-12-en-28-oic acid. This aglycone contains a C-23 formyl group, which is thought to be the biosynthetic intermediate in the enzymatic oxidation of the C-23 hydroxy group of bayogenin, eventually leading to the carboxylation of medicagenic acid (Figure 4). ${ }^{31}$ Thus, compounds containing aglycone $\mathrm{A}$ as the sapogenin could be $2 \beta, 3 \beta$-dihydroxy-23-oxo-olean-12-en-28-oic acid glycosides. Another identified saponin yielded an aglycone anion at $\mathrm{m} / z 469$ (Figure 5D) and a predicted molecular formula of $\mathrm{C}_{30} \mathrm{H}_{46} \mathrm{O}_{4}$ (aglycone B). Similar to aglycone A, this compound may contain a C-23 formyl group and may be the result of an enzymatic oxidation of hederagenin, leading to $3 \beta$-hydroxy-23-oxo-olean-12-en-28-oic acid (Figure 4).
Aglycone C, a third unknown aglycone encountered in one of the saponins of $M$. truncatula hairy roots, yielded a first product ion at $m / z$ 437. Similar to medicagenic acid, this ion is the result of the decarboxylation and dehydration of aglycone $\mathrm{C}$, which has a calculated molecular formula of $\mathrm{C}_{30} \mathrm{H}_{44} \mathrm{O}_{6}$. Decarboxylation and dehydration of aglycones are observed only in medicagenic $\operatorname{acid}^{2}$ and zanhic acid ${ }^{8}$ and, thus, could be related to the presence of a C-23 carboxylic group on the aglycone. Consequently, we assume that aglycone $\mathrm{C}$ contains a $\mathrm{C}-23$ carboxylic group. Two saponins contain an aglycone (aglycone D) yielding an anion at $\mathrm{m} / \mathrm{z} 489$ (Figure 1C) and a calculated molecular formula of $\mathrm{C}_{30} \mathrm{H}_{50} \mathrm{O}_{5}$. No aglycones with this molecular formula have been described in Medicago to date, but, assuming a $\beta$-amyrin-derived triterpene skeleton, the molecular formula points toward an aglycone containing five hydroxy groups. Thus, we postulate that aglycone $\mathrm{D}$ could be an oxidation product of soyasapogenol A. However, since we cannot postulate a structure based on these assumptions, we did not include the aglycones $\mathrm{C}$ and $\mathrm{D}$ in Figure 3.

\section{EXPERIMENTAL SECTION}

Generation and Cultivation of Transgenic M. truncatula Hairy Roots. A. rhizogenes-mediated transformation of $M$. truncatula (ecotype Jemalong J5) hairy roots was carried out as reported ${ }^{20}$ with the following modifications. After scarification by treatment with sulfuric acid for $5 \mathrm{~min}$, seeds were surface sterilized with $12 \% \mathrm{NaOCl}$ during 2 min and washed with sterile $\mathrm{H}_{2} \mathrm{O}$. Subsequently, seeds were treated with $1 \mu \mathrm{M}$ 6-benzylaminopurine for $3 \mathrm{~h}$ and thereafter allowed to germinate on wet, sterile $3 \mathrm{~mm}$ Whatman paper at room temperature in the dark. After 2 days of germination, the seedlings were wounded by cutting approximately $2 \mathrm{~mm}$ from the root tip of the radicle. The wounded seedlings were infected with $A$. rhizogenes and placed on slanted agar plates containing Murashige and Skoog (MS) medium ( $\mathrm{pH}$ 5.8) supplemented with vitamins (Duchefa). The plates were sealed with micropore tape and placed vertically. Cocultivation was allowed for 10 days under a $16 \mathrm{~h} / 8 \mathrm{~h}$ light $/$ dark regime at $22^{\circ} \mathrm{C}$. After 10 days, the plants were transferred to new plates, containing $100 \mathrm{mg} / \mathrm{L}$ cefotaxime to prevent $A$. rhizogenes growth, and incubated under identical conditions. After 10 days, hairy roots were excised from the plants and transferred to liquid MS medium ( $\mathrm{pH}$ 5.8) supplemented with vitamins, $1 \%(\mathrm{w} / \mathrm{v})$ sucrose, and $100 \mathrm{mg} / \mathrm{L}$ cefotaxime to eliminate $A$. rhizogenes contamination. The hairy roots were grown for 7 days in the dark at $24{ }^{\circ} \mathrm{C}$ and shaking at $300 \mathrm{rpm}$. Subsequently, the roots were transferred to horizontal Petri dishes containing solid MS medium ( $\mathrm{pH}$ 5.8) supplemented with $1 \%(\mathrm{w} / \mathrm{v})$ sucrose and $100 \mathrm{mg} / \mathrm{L}$ cefotaxime and grown in the dark at $24{ }^{\circ} \mathrm{C}$. After 3 weeks, young hairy root tissue was subcultured to solid medium without antibiotics. For maintenance, the 
A ctr2b RT: 27.28 AV: 1 NL: 1.16E3

T: ITMS - c ESI d Full ms2957.51@cid35.00 [250.00-970.00]

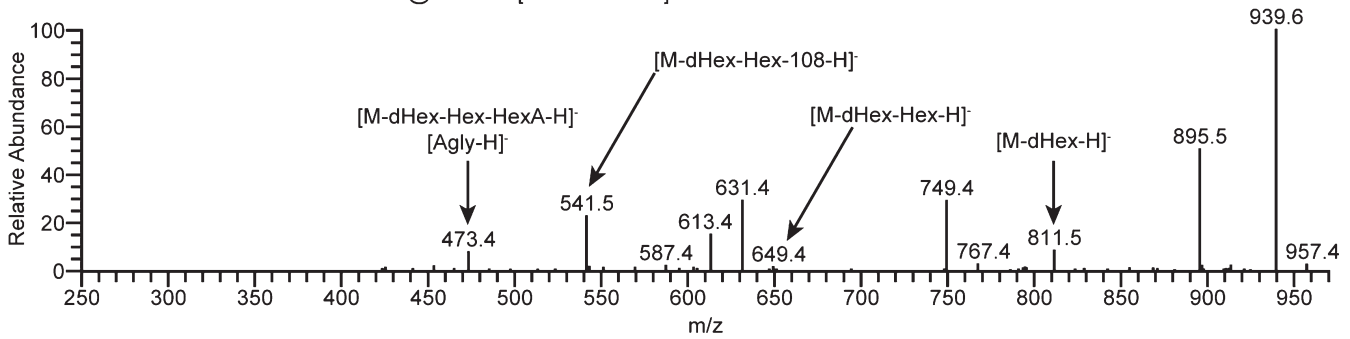

B ctr1b RT: 30.44 AV: 1 NL: 7.21E3

T: ITMS - c ESI d Full ms2 $957.51 @$ cid35.00 [250.00-970.00]

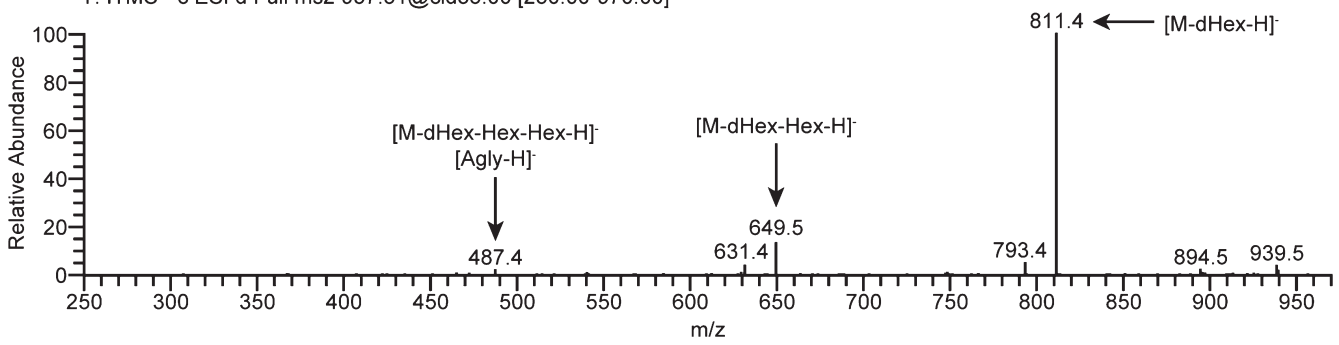

C ctr1b RT: 31.43 AV: 1 NL: $1.40 \mathrm{E} 3$

T: ITMS - c ESI d Full ms2 955.49@cid35.00 [250.00-970.00]

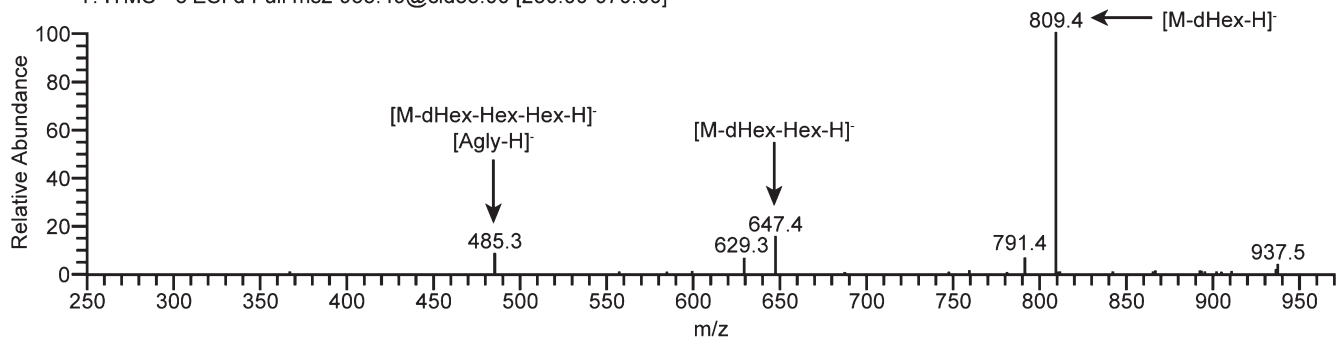

D ctr2b RT: 32.44 AV: 1 NL: $1.57 E 3$

T: ITMS - c ESI d Full ms2 925.48@cid35.00 [240.00-940.00]

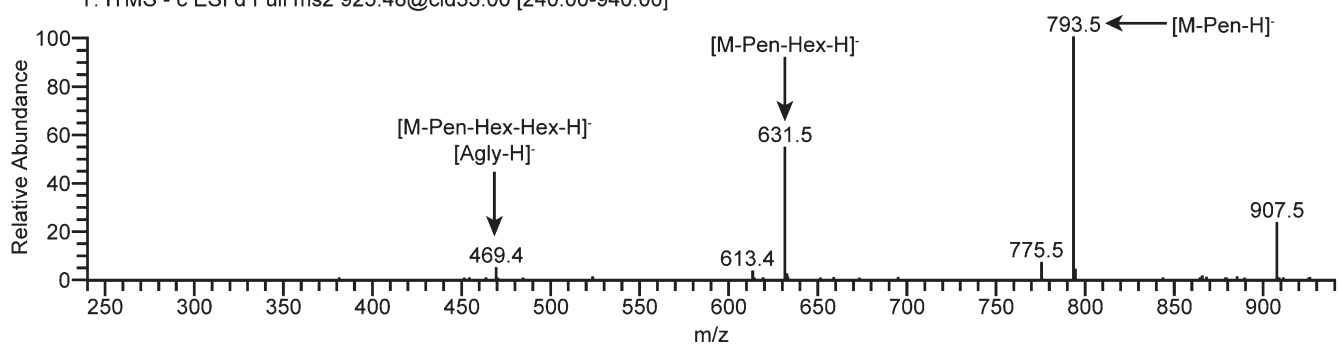

Figure 5. LC ESI FT-ICR MS chromatograms leading to the detection of the various aglycones. (A) $\mathrm{MS}^{2}$ fragmentation of dHex-Hex-HexAsoyasapogenol A. (B) MS ${ }^{2}$ fragmentation of dHex-Hex-Hex-bayogenin. (C) MS ${ }^{2}$ fragmentation of dHex-Hex-Hex-aglycone A. (D) MS ${ }^{2}$ fragmentation of Pen-Hex-Hex-aglycone B.

axenic hairy roots cultures were subcultured every 3 weeks onto fresh plates. Hairy roots for metabolic profiling were grown for 21 days in liquid MS medium ( $\mathrm{pH}$ 5.8) supplemented with vitamins and $1 \%(\mathrm{w} / \mathrm{v})$ sucrose.

Metabolite Extractions. M. truncatula hairy roots, grown for 21 days in liquid medium, were harvested and rinsed with purified $\mathrm{H}_{2} \mathrm{O}$ under vacuum filtration. Subsequently, the roots were frozen and ground in liquid $\mathrm{N}_{2}$. Of the ground material, $400 \mathrm{mg}$ was extracted with $1 \mathrm{~mL}$ of $\mathrm{MeOH}$ at room temperature for $10 \mathrm{~min}$, followed by centrifugation for $10 \mathrm{~min}$ at $20800 \mathrm{~g}$. Under vacuum, $500 \mu \mathrm{L}$ of the supernatant was evaporated to dryness, and the residue was dissolved in $600 \mu \mathrm{L}$ of $\mathrm{H}_{2} \mathrm{O}$ / cyclohexane $(2: 1, \mathrm{v} / \mathrm{v})$. Samples were centrifuged $(10 \mathrm{~min}$ at $20800 \mathrm{~g})$, and $200 \mu \mathrm{L}$ of the aqueous phase was retained for analysis.

LC ESI FT-ICR MS. For reversed-phase LC, an Acquity UPLC BEH C18 column $(150 \times 2.1 \mathrm{~mm}, 1.7 \mu \mathrm{m}$; Waters, Milford, MA $)$ was serially coupled to an Acquity UPLC BEH C18 column $(100 \times 2.1 \mathrm{~mm}, 1.7 \mu \mathrm{m})$ and mounted on an ultra-high-performance LC system consisting of a Accela pump (Thermo Electron Corporation, Waltham, MA, USA) and Accela autosampler (Thermo Electron Corporation). The Accela LC system was hyphenated to a LTQ FT Ultra (Thermo Electron Corporation) via an electrospray ionization source. The following gradient was run using water/MeCN (99:1, v/v) acidified with $0.1 \%$ (v/v) HOAc (solvent A) and $\mathrm{MeCN} /$ water (99:1, v/v) acidified with $0.1 \%$ (v/v) HOAc (solvent B): time 0 min, $5 \%$ B; 30 min, 55\% B; 35 min, $100 \% \mathrm{~B}$. The loop size, flow, and column temperature were $25 \mu \mathrm{L}$, $300 \mu \mathrm{L} / \mathrm{min}$, and $80^{\circ} \mathrm{C}$, respectively. Full loop injection was applied. Negative ionization was obtained with the following parameter values: capillary temperature $150{ }^{\circ} \mathrm{C}$, sheath gas 25 (arbitrary units), aux. gas 3 (arbitrary units), and spray voltage $4.5 \mathrm{kV}$. Full FT-MS spectra between $\mathrm{m} / z 120-1400$ were recorded at a resolution of 100000 . For identification, full MS spectra were interchanged with a dependent $\mathrm{MS}^{2}$ scan event in which the most abundant ion in the previous full MS scan was 
fragmented, and two dependent $\mathrm{MS}^{3}$ scan events in which the two most abundant daughter ions were fragmented. The collision energy was set at $35 \%$.

\section{AUTHOR INFORMATION}

\section{Corresponding Author}

${ }^{*}$ Tel: + 32 (0) 93313 851. Fax: + 32 (0) 93313 809. E-mail: alain.goossens@psb-vib.ugent.be.

\section{ACKNOWLEDGMENT}

The authors thank Martine De Cock for help in preparing the manuscript. This work was supported by the Agency for Innovation by Science and Technology in Flanders ("Strategisch Basisonderzoek" project SBO040093).

\section{REFERENCES}

(1) Dixon, R. A.; Sumner, L. W. Plant Physiol. 2003, 131, 878-885.

(2) Huhman, D. V.; Sumner, L. W. Phytochemistry 2002, 59, 347-360.

(3) Sparg, S. G.; Light, M. E.; van Staden, J. J. Ethnopharmacol. 2004, 94, 219-243.

(4) Sun, H. X.; Xie, Y.; Ye, Y. P. Vaccine 2009, 27, 1787-1796.

(5) Dixon, R. A.; Pasinetti, G. M. Plant Physiol. 2010, 154, 453-457.

(6) Farag, M. A.; Deavours, B. E.; de Fátima, A.; Naoumkina, M.; Dixon, R. A.; Sumner, L. W. Plant Physiol. 2009, 151, 1096-1113.

(7) Huhman, D. V.; Berhow, M. A.; Sumner, L. W. J. Agric. Food Chem. 2005, 53, 1914-1920.

(8) Kapusta, I.; Janda, B.; Stochmal, A.; Oleszek, W. J. Agric. Food Chem. 2005, 53, 7654-7660.

(9) Kapusta, I.; Stochmal, A.; Perrone, A.; Piacente, S.; Pizza, C.; Oleszek, W. J. Agric. Food Chem. 2005, 53, 2164-2170.

(10) Farag, M. A.; Huhman, D. V.; Lei, Z. T.; Sumner, L. W. Phytochemistry 2007, 68, 342-354.

(11) Schliemann, W.; Ammer, C.; Strack, D. Phytochemistry 2008, 69, 112-146.

(12) Georgiev, M. I.; Pavlov, A. I.; Bley, T. Appl. Microbiol. Biotechnol. 2007, 74, 1175-1185.

(13) Guillon, S.; Trémouillaux-Guiller, J.; Pati, P. K.; Rideau, M.; Gantet, P. Trends Biotechnol. 2006, 24, 403-409.

(14) Guillon, S.; Trémouillaux-Guiller, J.; Pati, P. K.; Rideau, M.; Gantet, P. Curr. Opin. Plant Biol. 2006, 9, 341-346.

(15) Sevón, N.; Oksman-Caldentey, K. M. Planta Med. 2002, $68,859-868$.

(16) Araújo, S. D.; Duque, A. S. R. L. A.; dos Santos, D. M. M. F.; Fevereiro, M. P. S. Plant Cell Tiss. Org. Cult. 2004, 78, 123-131.

(17) Chabaud, M.; Larsonneau, C.; Marmouget, C.; Huguet, T. Plant Cell Rep. 1996, 15, 305-310.

(18) Cook, D. R. Curr. Opin. Plant Biol. 1999, 2, 301-304.

(19) Trieu, A. T.; Burleigh, S. H.; Kardailsky, I. V.; MaldonadoMendoza, I. E.; Versaw, W. K.; Blaylock, L. A.; Shin, H.; Chiou, T. J.; Katagi, H.; Dewbre, G. R.; Weigel, D.; Harrison, M. J. Plant J. 2000, 22, 531-541.

(20) Boisson-Dernier, A.; Chabaud, M.; Garcia, F.; Bécard, G.; Rosenberg, C.; Barker, D. G. Mol. Plant Microbe Interact. 2001, 14, 695-700.

(21) Liu, S. Y.; Cui, M.; Liu, Z. Q.; Song, F. R.; Mo, W. J. J. Am. Soc. Mass Spectrom. 2004, 15, 133-141.

(22) Domon, B.; Costello, C. E. Glycoconj. J. 1988, 5, 397-409.

(23) Levigne, S. V.; Ralet, M. C. J.; Quéméner, B. C.; Pollet, B. N. L.; Lapierre, C.; Thibault, J. F. J. Plant Physiol. 2004, 134, 1173-1180.

(24) Quéméner, B.; Ralet, M. C. J. Mass Spectrom. 2004, 39, $1153-1160$.

(25) Vanholme, R.; Ralph, J.; Akiyama, T.; Lu, F. C.; Pazo, J. R.; Kim, H.; Christensen, J. H.; Van Reusel, B.; Storme, V.; De Rycke, R.; Rohde, A.; Morreel, K.; Boerjan, W. Plant J. 2010, 64, 885-897.
(26) Jin, M.; Yang, Y.; Su, B.; Ren, Q. J. Chromatogr. B 2007, $846,169-175$.

(27) Confalonieri, M.; Cammareri, M.; Biazzi, E.; Pecchia, P.; Fevereiro, M. P. S.; Balestrazzi, A.; Tava, A.; Conicella, C. Plant Biotechnol. J. 2009, 7, 172-182.

(28) Hu, Z. B.; Du, M. J. Integr. Plant Biol. 2006, 48, 121-127.

(29) Tava, A.; Avato, P. Nat. Prod. Commun. 2006, 1, 1159-1180.

(30) Naoumkina, M. A.; Modolo, L. V.; Huhman, D. V.; UrbanczykWochniak, E.; Tang, Y. H.; Sumner, L. W.; Dixon, R. A. Plant Cell 2010, 22, 850-866.

(31) Tava, A.; Scotti, C.; Avato, P. Phytochem. Rev. 2011, in press (DOI $10.1007 /$ s11101-010-9169-x). 